\title{
Representing some families of monotone maps by principal lattice congruences
}

\author{
GÁBor CZÉDLI
}

Dedicated to George Grätzer on the occasion of his eightieth birthday

\begin{abstract}
For a lattice $L$ with 0 and 1 , let $\operatorname{Princ}(L)$ denote the set of principal congruences of $L$. Ordered by set inclusion, it is a bounded ordered set. In 2013, G. Grätzer proved that every bounded ordered set is representable as $\operatorname{Princ}(L)$; in fact, he constructs $L$ as a lattice of length 5 . For $\{0,1\}$-sublattices $A \subseteq B$ of $L$, congruence generation defines a natural map $\operatorname{Princ}(A) \rightarrow \operatorname{Princ}(B)$. In this way, every family of $\{0,1\}$-sublattices of $L$ yields a small category of bounded ordered sets as objects and certain 0 -separating $\{0,1\}$-preserving monotone maps as morphisms such that every hom-set consists of at most one morphism. We prove the converse: every small category of bounded ordered sets with these properties is representable by principal congruences of selfdual lattices of length 5 in the above sense. As a corollary, we can construct a selfdual lattice $L$ in G. Grätzer's above-mentioned result.
\end{abstract}

\section{Introduction}

By an old result of N. Funayama and T. Nakayama [8], the congruence lattice $\operatorname{Con}(L)$ of a lattice $L$ is a distributive algebraic lattice. For finite lattices, the converse also holds: by a classical result of R. P. Dilworth, every finite distributive lattice $D$ can be represented as the congruence lattice of a finite lattice $L$; see [1], and see also G. Grätzer and E. T. Schmidt [22] for the first published proof. As surveyed in G. Grätzer [10], many improvements of this theorem yield an $L$ with strong additional properties; here we mention only G. Grätzer and E. Knapp [15], where $L$ is a finite rectangular (and, thus, planar and semimodular) lattice, G. Grätzer and E. T. Schmidt [23], where $L$ is rectangular and each of its congruences is principal, and G. Czédli and E. T. Schmidt [7], where $L$ is almost-geometric. If finiteness is dropped, then the theory of representability of a single lattice in the above sense culminated in F. Wehrung [29], where a non-representable distributive algebraic lattice $D$ was constructed; this $D$ has $\aleph_{\omega+1}$ compact elements. Later, P. Růžička [28] reduced $\aleph_{\omega+1}$ to $\aleph_{2}$; note that no further reduction is possible by A.P. Huhn [24].

Motivated by the rich history of congruence lattice representation problem, G. Grätzer in [12] has recently started an analogous new topic of lattice theory. Namely, for a lattice $L$, let $\operatorname{Princ}(L)=\langle\operatorname{Princ}(L) ; \subseteq\rangle$ denote the ordered set

2010 Mathematics Subject Classification: 06B10

December 23, 2016

Key words and phrases: principal congruence, lattice congruence, ordered set, order, poset, quasi-colored lattice, preordering, quasiordering, monotone map, categorified lattice, functor, lattice category, lifting diagrams (to appear in Algebra Universalis). 
of principal congruences of $L$. A congruence is principal if it is generated by a pair $\langle a, b\rangle$ of elements. Ordered sets (also called partially ordered sets or posets) and lattices with 0 and 1 are called bounded. If $L$ is a bounded lattice, then $\operatorname{Princ}(L)$ is a bounded ordered set. Conversely, by G. Grätzer [12], each bounded ordered set $P$ is isomorphic to $\operatorname{Princ}(L)$ for an appropriate bounded lattice $L$ of length 5. The ordered sets $\operatorname{Princ}(L)$ of countable lattices $L$ were characterized as directed countable ordered sets with 0 by G. Czédli [5].

There are many results representing a monotone map between two finite distributive lattices by congruence lattices; here we mention only G. Grätzer, H. Lakser [16], [17], and [18], G. Grätzer, H. Lakser, and E. T. Schmidt [19] and [21], and G. Czédli [2]; see G. Grätzer [10] for a survey again. Motivated by these results and G. Grätzer in [12], G. Czédli [3] represents two bounded ordered sets and a certain map between them by principal lattice congruences simultaneously; see Proposition 2.1 later.

In this paper, we give a simultaneous representation for a set of bounded ordered sets together with some collection of monotone maps by principal lattice congruences. Even the result of G. Grätzer [12] and that of [3] are strengthened, because we construct selfdual lattices of length 5 .

1.1. Outline. In Section 2, we formulate the main result of the paper, Theorem 2.8. Also, Proposition 2.1 and Example 2.2 discuss two particular cases; they help in understanding quickly what Theorem 2.8 asserts. Based on Figures 1, 2, 3, 4 and Example 3.1, Section 3 motivates the main ideas of the proof without rigorous details. In Section 4, we construct some lattices, and we prove Lemma 4.6 stating that they are quasi-colored lattices. Also, Lemma 4.7 determines the ordered sets of principal congruences of our quasi-colored lattices. Based on Section 4, Section 5 completes the proof of Theorem 2.8. Finally, Section 6 is devoted to some concluding remarks; in particular, we point out how one can construct smaller lattices.

\section{Our result}

2.1. Representing one monotone map. Given two bounded ordered sets, $P$ and $Q$, a map $\psi: P \rightarrow Q$ is called a $\{0,1\}$-preserving monotone map if $\psi\left(0_{P}\right)=0_{Q}, \psi\left(1_{P}\right)=1_{Q}$, and, for all $x, y \in P, x \leq_{P} y$ implies that $\psi(x) \leq_{Q}$ $\psi(y)$. If, in addition, $0_{P}$ is the only preimage of $0_{Q}$, that is, if $\psi^{-1}\left(0_{Q}\right)=\left\{0_{P}\right\}$, then we say that $\psi$ is a 0 -separating $\{0,1\}$-preserving monotone map. Note that monotone maps are also called order-preserving maps. For a lattice $L$ and $x, y \in L$, the principal congruence generated by $\langle x, y\rangle$ is denoted by con $(x, y)$ or $\operatorname{con}_{L}(x, y)$. Similarly, for $X \subseteq L^{2}$, the least congruence including $X$ is denoted by $\operatorname{con}_{L}(X)$. If $L_{0}$ is a $\{0,1\}$-sublattice of $L_{1}$, then the natural extension map

$$
\zeta_{L_{0}, L_{1}}: \operatorname{Princ}\left(L_{0}\right) \rightarrow \operatorname{Princ}\left(L_{1}\right) \text { defined by } \operatorname{con}_{L_{0}}(x, y) \mapsto \operatorname{con}_{L_{1}}(x, y)
$$


is clearly a 0 -separating $\{0,1\}$-preserving monotone map. (It is well defined, because $\zeta_{L_{0}, L_{1}}\left(\operatorname{con}_{L_{0}}(x, y)\right)$ is clearly the same as $\operatorname{con}_{L_{1}}\left(\operatorname{con}_{L_{0}}(x, y)\right)$.) We know from $G$. Czédli [3] that each 0 -separating $\{0,1\}$-preserving monotone map between two bounded ordered sets is of the form (2.1) in a reasonable sense. More exactly, with the convention that we compose maps from right to left, we have the following statement.

Proposition 2.1 (G. Czédli [3]). Let $\left\langle P_{0} ; \leq_{0}\right\rangle$ and $\left\langle P_{1} ; \leq_{1}\right\rangle$ be bounded ordered sets. If $\psi$ is a 0 -separating $\{0,1\}$-preserving monotone map from $\left\langle P_{0} ; \leq_{0}\right\rangle$ to $\left\langle P_{1} ; \leq_{1}\right\rangle$, then there exist a bounded lattice $L_{1}, a\{0,1\}$-sublattice $L_{0}$ of $L_{1}$, and order isomorphisms

$$
\xi_{0}:\left\langle P_{0} ; \leq_{0}\right\rangle \rightarrow\left\langle\operatorname{Princ}\left(L_{0}\right) ; \subseteq\right\rangle \quad \text { and } \quad \xi_{1}:\left\langle P_{1} ; \leq_{1}\right\rangle \rightarrow\left\langle\operatorname{Princ}\left(L_{1}\right) ; \subseteq\right\rangle
$$

such that $\psi=\xi_{1}^{-1} \circ \zeta_{L_{0}, L_{1}} \circ \xi_{0}$; that is, the diagram

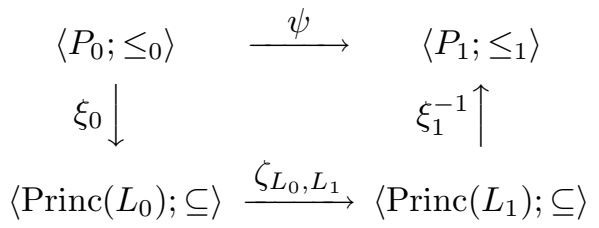

is commutative.

Therefore, 0 -separating $\{0,1\}$-preserving monotone maps between two ordered sets are characterized up to isomorphism as extension maps (2.1) for principal lattice congruences.

2.2. Simultaneous representation of many monotone maps. A lattice is of length 5 if it has a 6 -element chain but does not have a 7 -element chain. Such a lattice is necessarily bounded. If $L_{1}$ is a lattice of length 5 , then it has many $\{0,1\}$-sublattices in general, and for any two comparable $\{0,1\}$ sublattices $L_{2} \subseteq L_{3}$ of $L_{1}$, the extension map $\zeta_{L_{2}, L_{3}}$ defined as in (2.1) is a 0 -separating $\{0,1\}$-preserving monotone map. This motivates the extension of Proposition 2.1 from a single monotone map $\psi$ to a family of such maps. First, we outline our purpose with an example.

Example 2.2. Let $S=\langle S ; \leq\rangle$ be the ordered index set in Figure 1 and, for each $i \in S$, let $\left\langle P_{i} ; \nu_{i}\right\rangle$ be the bounded ordered set given in the figure. Furthermore, for every $i \prec j$ in $S$, let $\psi_{i j}$ be the 0 -separating $\{0,1\}$-preserving monotone map $\psi_{i j}: P_{i} \rightarrow P_{j}$ indicated by dotted curves. The obvious images of 0 and 1 are not indicated on purpose. For $i<j$ but $i \nprec j, \psi_{i j}$ is also defined by the rule $\psi_{01}=\psi_{21} \circ \psi_{02}=\psi_{31} \circ \psi_{03}$. Our goal is to find a selfdual lattice $L_{1}$ of length 5 and selfdual $\{0,1\}$-sublattices $L_{0}, L_{2}, L_{3}$ of $L_{1}$ such that $\left\langle P_{i} ; \nu_{i}\right\rangle \cong \operatorname{Princ}\left(L_{i}\right)$ and $\psi_{i j}$ is represented by $\zeta_{L_{i}, L_{j}}$ for all $i<j$ in the same sense as $\psi:=\psi_{01}$ is represented in (2.2). 

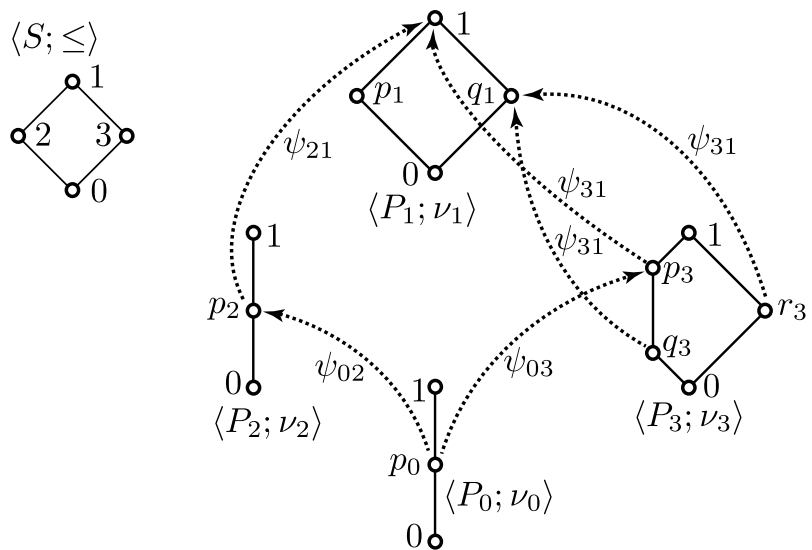

Figure 1. Monotone maps to represent; see Example 2.2

To give an exact description of our goal, the most economic way is to use the rudiments of category theory. First, we define some concrete categories and functors. An ordered set is nontrivial if it has at least two elements.

\section{Notation and definition 2.3.}

(i) As usual, we often consider an ordered set $S=\langle S ; \leq\rangle$ a small category. This category, denoted by $\operatorname{Cat}(S)$ or $\operatorname{Cat}(\langle S ; \leq\rangle)$, consists of the elements of $S$ as objects and the pairs belonging to the ordering relation $\leq$ as morphisms.

(ii) The category of nontrivial bounded ordered sets with 0 -separating $\{0,1\}$ preserving monotone maps will be denoted by $\mathbf{P O S}_{01}^{0 \mathrm{~s}}$.

(iii) The category of selfdual lattices of length 5 with lattice embeddings as morphisms will be denoted by $\mathbf{L a t}_{\mathrm{sd} 5}^{\mathrm{emb}}$.

(iv) We define a functor Princ: $\mathbf{L a t}_{\mathrm{sd} 5}^{\mathrm{emb}} \rightarrow \mathbf{P O S}_{01}^{0 \mathrm{~s}}$ as follows. For an object, that is, a lattice $L$ in $\mathbf{L a t}_{\mathrm{sd} 5}^{\mathrm{emb}}, \operatorname{Princ}(L)=\langle\operatorname{Princ}(L) ; \subseteq\rangle$ is the ordered set of principal congruences of $L$. For a morphism $f: K \rightarrow L$ in $\mathbf{L a t}_{\mathrm{sd} 5}^{\mathrm{emb}}$, we let

$$
\begin{aligned}
\operatorname{Princ}(f): & \operatorname{Princ}(K) \rightarrow \operatorname{Princ}(L), \text { defined by } \\
& \operatorname{con}_{K}(x, y) \mapsto \operatorname{con}_{L}(f(x), f(y)) .
\end{aligned}
$$

Note that every morphism in $\mathbf{L a t}_{\mathrm{sd} 5}^{\mathrm{emb}}$ is a cover-preserving and $\{0,1\}$-preserving lattice embedding. It is straightforward to see that $\operatorname{Princ}(f)\left(\operatorname{con}_{K}(x, y)\right)$ is the same as

$$
\operatorname{con}_{L}\left(\left\{\langle f(u), f(v)\rangle:\langle u, v\rangle \in \operatorname{con}_{K}(x, y)\right\}\right) .
$$

Hence, the choice of $x$ and $y$ in (2.3) is irrelevant, and Princ $(f)$ is a well-defined map. It is clearly 0 -separating and monotone. Since $K$ is a $\{0,1\}$-sublattice of $L$, Princ $(f)$ is $\{0,1\}$-preserving. So, $\operatorname{Princ}(f)$ is a morphism in $\mathbf{P O S}_{01}^{0 \text { s }}$. It is easy to see that Princ: $\mathbf{L a t}_{\mathrm{sd}}^{\mathrm{emb}} \rightarrow \mathbf{P O S}_{01}^{0 \mathrm{~s}}$ is a functor. 
Remark 2.4. If $K$ is a $\{0,1\}$-sublattice of $L$ and $f: K \rightarrow L$ is the inclusion map, then $\operatorname{Princ}(f)$ is the same as $\zeta_{K, L}$ given in (2.1).

Remark 2.5. We have excluded the singleton ordered sets from $\mathbf{P O S}_{01}^{0 \mathrm{~s}}$. This is not a serious restriction, because the only arrow starting from or departing at a singleton ordered set in $\mathbf{P} \mathbf{O S}_{01}^{0 \mathrm{~s}}$ is an isomorphism between two singleton ordered sets. On the other hand, for a lattice $L,|\operatorname{Princ}(L)|=1$ iff $|L|=1$, which is a non-interesting case.

Definition 2.6. Let $S$ be an ordered set and let $F: \operatorname{Cat}(S) \rightarrow \mathbf{P O S}_{01}^{0 \mathrm{~s}}$ be a functor. Following P. Gillibert and F. Wehrung [9], we say that a functor

$$
E: \operatorname{Cat}(S) \rightarrow \mathbf{L a t}_{\mathrm{sd} 5}^{\mathrm{emb}}
$$

lifts $F$ with respect to the functor Princ, if $F$ is naturally isomorphic (also called naturally equivalent) to the composite functor Princ $\circ E$. We say that $F$ is representable by principal lattice congruences in $\mathbf{L} \mathbf{a t}_{\mathrm{sd} 5}^{\mathrm{emb}}$ if there exists a functor $E: \mathbf{C a t}(S) \rightarrow \mathbf{L a t}_{\mathrm{sd} 5}^{\mathrm{emb}}$ that lifts $F$ with respect to Princ.

As opposed to category theorists, an algebraist may feel that a family of not necessarily distinct lattices together with embeddings is not as nice as it should be. Hence, we also introduce the following concept.

Definition 2.7. We say that $F: \operatorname{Cat}(S) \rightarrow \mathbf{P O S}_{01}^{0 \mathrm{~s}}$ from Definition 2.6 is concretely representable by principal lattice congruences in $\mathbf{L a t}_{\mathrm{sd} 5}^{\mathrm{emb}}$ if there are a lattice $L$ in $\mathbf{L a t}_{\mathrm{sd} 5}^{\mathrm{emb}}$ and a functor $E: \mathbf{C a t}(S) \rightarrow \mathbf{L a t}_{\mathrm{sd} 5}^{\mathrm{emb}}$ such that

(i) for every $s \in S, E(s)$ is a $\{0,1\}$-sublattice of $L$;

(ii) for every "arrow" $s \leq t$ of $\mathbf{C a t}(S), E(s)$ is a $\{0,1\}$-sublattice of $E(t)$ and $E(s \leq t)$ is the inclusion map from $E(s)$ into $E(t)$;

(iii) for every $s, t \in S$, if $E(s) \subseteq E(t)$, then $s \leq t$; and

(iv) $E$ lifts $F$ with respect to Princ.

In case of concrete representability, Remark 2.4 simplifies the situation, since the functor Princ is applied only for inclusion maps. Clearly, if $F$ from Definition 2.7 is concretely representable by principal congruences, then it is representable by principal congruences. Our main result is the following.

Theorem 2.8. For every ordered set $S$, every functor

$$
F: \mathbf{C a t}(S) \rightarrow \mathbf{P O S}_{01}^{0 \mathrm{~s}}
$$

is concretely representable by principal lattice congruences in $\mathbf{L a t}_{\mathrm{sd} 5}^{\mathrm{emb}}$.

P. Gillibert and F. Wehrung [9, page 12] points out that a functor can seldom be represented (that is, lifted). The representability of some examples mentioned in [9, page 12] never happens for trivial reasons. Hence, it is not a surprise that the proof of Theorem 2.8 in this paper is not short.

To show the strength of Theorem 2.8, we make two observations. First, observe that Proposition 2.1 follows from the particular case of the Theorem where $S$ is the two-element chain. Second, applying the theorem for the 
case $|S|=1$, we obtain the following generalization of the main result of $\mathrm{G}$. Grätzer [12].

Corollary 2.9. Every nontrivial bounded ordered set $P$ is isomorphic to the ordered set of principal congruences of some selfdual lattice $L$ of length 5 .

It will be clear from our construction that for a finite $P$ in Corollary 2.9, we always have a finite selfdual lattice $L$ of length 5 . Similarly, if $S$ in Theorem 2.8 is finite and so is $F(s)$ for every $s \in S$, then $F$ can be lifted by a functor $E: \operatorname{Cat}(S) \rightarrow \mathbf{L a t}_{\mathrm{sd} 5}^{\mathrm{emb}}$ with respect to Princ such that $E(s)$ is a finite lattice for every $s \in S$.

2.3. Added on May 4, 2016. One of the referees has pointed out that our construction and proof yield a little more than stated in Theorem 2.8. Following M. Kamara [25], a polarity lattice is a structure $\langle L ; \vee, \wedge, \pi\rangle$ such that $\langle L ; \vee, \wedge\rangle$ is a lattice and $\pi$ is a polarity, that is, is a unary operation satisfying the identities

$$
\pi(\pi(x))=x, \quad \pi(x \vee y)=\pi(x) \wedge \pi(y), \text { and } \pi(x \wedge y)=\pi(x) \vee \pi(y) .
$$

Clearly, selfdual lattices are exactly the lattice reducts of polarity lattices. We are interested in polarity lattices $\langle L ; \vee, \wedge, \pi\rangle$ satisfying the property

$$
\operatorname{Princ}(\langle L ; \vee, \wedge, \pi\rangle)=\operatorname{Princ}(\langle L ; \vee, \wedge\rangle) \text { and length }(\langle L ; \vee, \wedge\rangle)=5 .
$$

Since every congruence is a join of principal congruences, the first equality in (2.4) is equivalent to the condition that every congruence of $\langle L ; \vee, \wedge\rangle$ is also a congruence of $\langle L ; \vee, \wedge, \pi\rangle$. Let $\mathbf{P} \mathbf{L a t}_{(2.4)}^{\mathrm{emb}}$ denote the category of polarity lattices satisfying (2.4) with embeddings as morphisms. (Embeddings are lattice embeddings commuting with $\pi$.) We can consider Princ a PLat $\mathbf{P}_{(2.4)}^{\mathrm{emb}} \rightarrow \mathbf{P O S}_{01}^{0 \mathrm{~s}}$ functor; see (2.3). Replacing Lat $\mathbf{s}_{\mathrm{sd} 5}^{\mathrm{emb}}$ with PLat $\mathbf{P}_{(2.4)}^{\mathrm{emb}}$ in Definitions 2.6 and 2.7, we obtain the concept of representability by principal congruences in $\mathbf{P L a t}_{(2.4)}^{\mathrm{emb}}$.

Addendum to Theorem 2.8 (Observed by an anonymous referee). The functor $F$ from Theorem 2.8 is concretely representable by principal congruences also in $\mathbf{P L a t}_{(2.4)}^{\mathrm{emb}}$.

At appropriate places, we will point out why $\pi$ is preserved and why our constructs are in $\mathbf{P L a t}_{(2.4)}^{\mathrm{emb}}$; this is sufficient to verify the Addendum.

Corollary 2.10. For every nontrivial bounded ordered set $P$, there exists a polarity lattice $\langle L ; \vee, \wedge, \pi\rangle \in \mathbf{P L a t}_{(2.4)}^{\mathrm{emb}}$ such that $P \cong \operatorname{Princ}(\langle L ; \vee, \wedge, \pi\rangle)$.

\section{Method and outline}

Our approach has three key ingredients. First, we borrow the basic idea of G. Grätzer [12] but our gadget lattice is different; see Remark 4.3 later.

Second, we use two recent results from G. Grätzer [13] and [14], which allow us to work with lattice congruences efficiently. 
Third, we need the quasi-coloring technique introduced in G. Czédli [2] and developed further in G. Czédli [5] and [3].

Due to some powerful lemmas from [5], the proof of Proposition 2.1 in [3] was quite short. As opposed to [3], the most involved lemmas from [5] cannot be used here directly, because the lattices in [5] are neither selfdual, nor of length 5. Hence, the present paper is much more self-contained than [3].

A quasiordered set is a structure $\langle H ; \nu\rangle$ where $H \neq \varnothing$ is a set and $\nu \subseteq H^{2}$ is a reflexive, transitive relation on $H$. Quasiordered sets are also called preordered sets. Instead of $\langle x, y\rangle \in \nu$, we often write $x \leq_{\nu} y$. Also, we write $x<_{\nu} y$ and $x \|_{\nu} y$ for the conjunction of $x \leq_{\nu} y$ and $y \not_{\nu} x$, and for the conjunction of $\langle x, y\rangle \notin \nu$ and $\langle y, x\rangle \notin \nu$, respectively. Similarly, $x={ }_{\nu} y$ will stand for the conjunction of $x \leq_{\nu} y$ and $y \leq_{\nu} x$. If $g \in H$ and $x \leq_{\nu} g$ for all $x \in H$, then $g$ is a greatest element of $H$; least elements are defined dually. They are not necessarily unique; if they are, then they are denoted by $1=1_{H}$ and $0=0_{H}$. In this case, we often use the notation

$$
H^{-01}=H \backslash\left\{0_{H}, 1_{H}\right\} .
$$

Given $H \neq \varnothing$, the quasiorderings on $H$ form a complete lattice with respect to set inclusion. For $X \subseteq H^{2}$, the least quasiorder on $H$ that includes $X$ is denoted by $\mathrm{quo}_{H}(X)$ or quo $(X)$. We write quo $(x, y)$ instead of quo $(\{\langle x, y\rangle\})$.

Next, in order to outline the construction needed in the proof of Theorem 2.8, we continue Example 2.2; see also Figure 1.

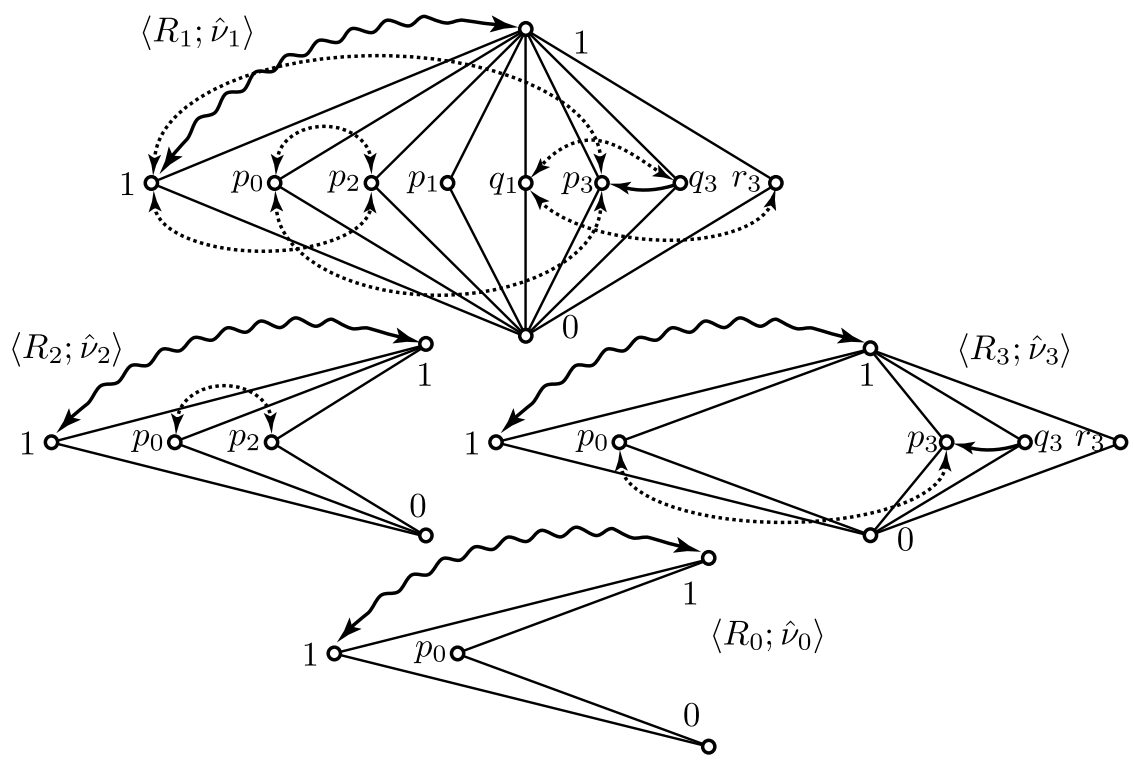

Figure 2. The quasiordered sets for Examples 2.2 and 3.1

Example 3.1 (Continuation of Example 2.2). 
(A) For the ordered sets $P_{i}$ in Figure 1, we assume that $P_{i} \cap P_{j}=\{0,1\}$ for $i \neq j \in S$. Define $R_{i}=\bigcup\left\{P_{j}: j \leq_{S} i\right\}$, for $i \in S$, see Figure 2 . Observe that, for $j \leq k \leq i, \nu_{j}$ and $\psi_{j k}=\left\{\langle x, y\rangle: x \in P_{j}, \psi_{j k}(x)=y\right\}$ are both relations on $R_{i}$. Let us agree that $\psi_{j j}$ is the identity map on $P_{j}$ and $\psi_{j k}^{-1}=\left\{\langle x, y\rangle: \psi_{j k}(y)=x\right\}$. So, for $i \in S$, we can let

$$
\hat{\nu}_{i}=\mathrm{quo}_{R_{i}}\left(\bigcup\left\{\nu_{j}: j \leq_{S} i\right\} \cup \bigcup\left\{\psi_{j k} \cup \psi_{j k}^{-1}: j \leq_{S} k \leq_{S} i\right\}\right) .
$$

In Figure 2, we give the quasiordered sets $\left\langle R_{i} ; \hat{\nu}_{i}\right\rangle$ as directed graphs; however, we do it in an unusual way. Namely, for each $i$, we depict $1 \in R_{i}$ twice, so the wavy arcs stand for equality. For example, $\left|R_{0}\right|=3$ but its graph contains 4 vertices. The duplicate vertices for 1 will serve explanatory purposes later. The graphs in Figure 2 contain arcs, that is, curved edges, and straight edges. The straight edges are understood as up-directed edges and they correspond to the meaning of 0 and 1 in $\left\langle P_{j} ; \nu_{j}\right\rangle$. The solid (non-wavy) directed arcs correspond to the orderings $\nu_{j}$. Whenever $y=\psi_{j k}(x)$ and $j \leq k \leq i$, then $R_{i}$ in Figure 2 contains the dotted directed $\operatorname{arcs}\langle x, y\rangle$ and $\langle y, x\rangle$; to make the figure less crowded, we use a single arc directed in both ways. Furthermore, we omit the dotted directed arcs of the forms $\langle 0,0\rangle$ and $\langle 1,1\rangle$. (Since the $\psi_{j k}$ are always $\{0,1\}$-preserving, these omitted arcs carry no information.) Note that the dotted arcs are inherited from Figure 1 but now they are directed in both ways. In this way, the $R_{i}$ in the figure are directed graphs and the $\hat{\nu}_{i}$ are the quasiorders generated by these graphs.

If $\langle H ; \nu\rangle$ is a quasiordered set, then $\Theta_{\nu}=\nu \cap \nu^{-1}$, also denoted by $={ }_{\nu}$, is known to be an equivalence relation, and the definition

$$
[x] \Theta_{\nu} \leq[y] \Theta_{\nu} \Longleftrightarrow x \leq_{\nu} y
$$

turns the quotient set $H / \Theta_{\nu}$ into an ordered set $\left\langle H / \Theta_{\nu} ; \leq\right\rangle$. In our case, it is clear from the figure that $\left\langle P_{i} ; \nu_{i}\right\rangle \cong\left\langle R_{i} / \Theta_{\hat{\nu}_{i}} ; \leq\right\rangle$ for $i \in S$. Furthermore, all we need to know about the $\psi_{j k}$, for $j \leq k \leq i$, is "encoded" in the quasiordered set $\left\langle R_{i} ; \hat{\nu}_{i}\right\rangle$.

(B) Next, we turn the quasiordered sets $\left\langle R_{i} ; \hat{\nu}_{i}\right\rangle$ of Figure 2 into lattices $W_{i}$ as follows. For every $u \neq 0$ in the "middle layer" of $\left\langle R_{i} ; \hat{\nu}_{i}\right\rangle$, we replace $u$ by a covering pair $a_{u} \prec b_{u}$. The duplicate of 1 in the middle layer is replaced by

$$
\text { a selfdual simple lattice } M \text { of length five such as } M=M_{4 \times 3}
$$

in Figure 9, which we will use later. We omit the wavy arcs and, usually,

$$
\text { we omit the arcs of the form }\langle u, 1\rangle \text {. }
$$

In $M$, we pick a covering pair $a_{1} \prec b_{1}$ such that a dual automorphism of $M$ maps $a_{1}$ to $b_{1}$. The lattices we obtain at this stage are depicted in Figure 3. Besides giving the lattice structures by straight lines, Figure 3 also contains the non-wavy arcs inherited from Figure 2, but we disregard them at present. For each $i \in S, W_{i}$ is a $\{0,1\}$-preserving sublattice of $W_{1}$. Observe that $\operatorname{Princ}\left(W_{i}\right)$ 
is a modular lattice of length 2 with pairwise distinct atoms $\operatorname{con}\left(a_{u}, b_{u}\right), u \in$ $R_{i} \backslash\{0,1\}$.

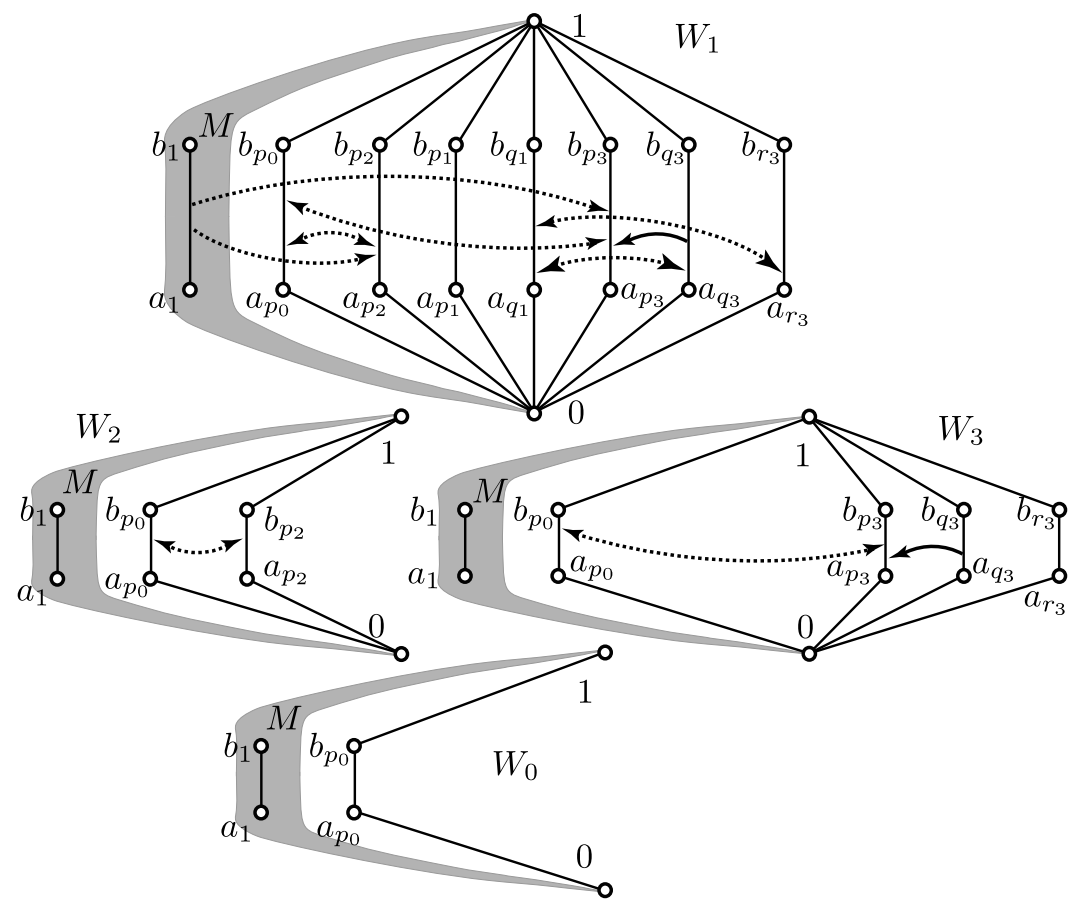

Figure 3. Auxiliary lattices with arcs for Examples 2.2 and 3.1

(C) From $W_{i}$ in Figure 3, we obtain our lattices $L_{i}, i \in S$, as follows. First, we change the remaining arcs among the vertices of Figure 2 to directed arcs among the corresponding "middle layer" edges in Figure 3. Next, whenever $\left\langle\left[a_{p}, b_{p}\right],\left[a_{q}, b_{q}\right]\right\rangle$ is a directed arc, we glue the selfdual lattice $G^{\mathrm{db}}(p, q)$ given in Figure 4 into $W_{i}$ in the natural way suggested by the notation, that is, we form $W_{i} \cup G^{\mathrm{db}}(p, q)$ such that $W_{i} \cap G^{\mathrm{db}}(p, q)=\left\{0, a_{p}, b_{p}, a_{q}, b_{q}, 1\right\}$. That is, for each directed arc in Figure 3, we add 22 new elements to $W_{i}$. The role of these 22 elements, which are black-filled in Figure 4 , is to force $\operatorname{con}\left(a_{p}, b_{p}\right) \leq$ $\operatorname{con}\left(a_{q}, b_{q}\right)$. In this way, after replacing all directed arcs by appropriate copies of the lattice from Figure 4, we obtain the lattices $L_{i}, i \in S$. Clearly, for $i \in S$, $L_{i}$ is a selfdual lattice of length 5 and it is a sublattice of $L_{1}$. Observe that $\left|W_{1}\right|=|M|+14=\left|M_{4 \times 3}\right|+14=28$ and $W_{1}$ has 11 directed arcs. (Those oriented in two ways count twice.) Hence, $\left|L_{1}\right|=28+11 \cdot 22=270$. Similarly, $\left|L_{0}\right|=14+2=16,\left|L_{2}\right|=14+4+2 \cdot 22=62$, and $\left|L_{3}\right|=14+8+3 \cdot 22=88$. In Remarks 6.1-6.2 and Example 6.3, we will point out how to obtain smaller lattices. 

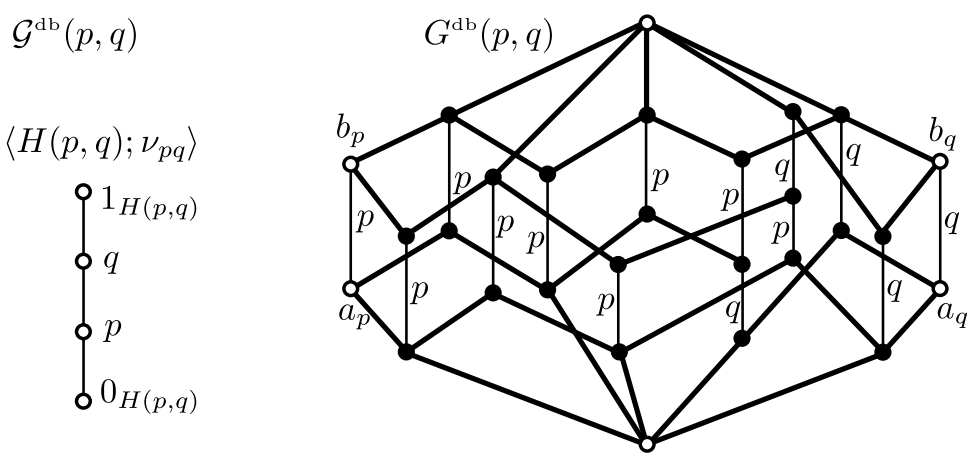

Figure 4 . The double gadget, $\mathcal{G}^{\mathrm{db}}(p, q)$

Even in Examples 2.2 and 3.1, it is not trivial that our 270-element lattice has only four principal congruences. In the rest of the paper, we give the general construction and prove that it works.

\section{The general construction and its properties}

4.1. Quasi-colored lattices. Let $L=\langle L ; \leq\rangle$ be an ordered set or a lattice. For $x, y \in L,\langle x, y\rangle$ is called an ordered pair of $L$ if $x \leq y$; this concept is consistent with the one used in previous work with quasi-colorings. An ordered pair $\langle x, y\rangle$ is a trivial ordered pair if $x=y$. The set of ordered pairs of $L$ is denoted by Pairs $\leq(L)$. If $X \subseteq L$, then Pairs $\leq(X)$ will stand for $X^{2} \cap$ Pairs $\leq(L)$. Note that we shall often use the fact that Pairs $\leq(S) \subseteq$ Pairs $\leq(L)$ holds for subsets $S$ of $L$; this explains why we work with ordered pairs rather than intervals. Note also that $\langle a, b\rangle$ is an ordered pair iff $b / a$ is a quotient. If $a \prec b$, then $\langle a, b\rangle$ is a covering pair. The set of covering pairs of $L$ is denoted by Pairs ${ }^{\prec}(L)$; note that Pairs ${ }^{\prec}(L) \subseteq$ Pairs $^{\leq}(L)$.

By a quasi-colored lattice we mean a structure $\mathcal{L}=\langle L, \leq ; \gamma ; H, \nu\rangle$ where $\langle L ; \leq\rangle$ is a lattice, $\langle H ; \nu\rangle$ is a quasiordered set, $\gamma$ : Pairs $\leq(L) \rightarrow H$ is a surjective map, and for all $\left\langle u_{1}, v_{1}\right\rangle,\left\langle u_{2}, v_{2}\right\rangle \in$ Pairs $^{\leq}(L)$,

(C1) if $\gamma\left(\left\langle u_{1}, v_{1}\right\rangle\right) \leq_{\nu} \gamma\left(\left\langle u_{2}, v_{2}\right\rangle\right)$, then $\operatorname{con}\left(u_{1}, v_{1}\right) \leq \operatorname{con}\left(u_{2}, v_{2}\right)$;

(C2) if $\operatorname{con}\left(u_{1}, v_{1}\right) \leq \operatorname{con}\left(u_{2}, v_{2}\right)$, then $\gamma\left(\left\langle u_{1}, v_{1}\right\rangle\right) \leq_{\nu} \gamma\left(\left\langle u_{2}, v_{2}\right\rangle\right)$.

This concept is taken from G. Czédli [2] and [5]. By the "antichain variant" of $(\mathrm{C} i)$ we mean the condition obtained from $(\mathrm{C} i)$ by substituting the equality sign for $\leq_{\nu}$ and $\leq$. Prior to [2], the name "coloring" was used for surjective maps satisfying the antichain variant of $(\mathrm{C} 2)$ in G. Grätzer, H. Lakser, and E.T. Schmidt [20], and for surjective maps satisfying the antichain variant of (C1) in G. Grätzer [10, page 39]. Note that in [2], [10], and [20], $\gamma(\langle u, v\rangle)$ was defined only for covering pairs $u \prec v$. To emphasize that $\operatorname{con}\left(u_{1}, v_{1}\right)$ and $\operatorname{con}\left(u_{2}, v_{2}\right)$ belong to the ordered $\operatorname{set} \operatorname{Princ}(L)$, we usually write $\operatorname{con}\left(u_{1}, v_{1}\right) \leq$ $\operatorname{con}\left(u_{2}, v_{2}\right)$ rather than $\operatorname{con}\left(u_{1}, v_{1}\right) \subseteq \operatorname{con}\left(u_{2}, v_{2}\right)$. It follows easily from (C1), 
(C2), and the surjectivity of $\gamma$ that if $\langle L, \leq ; \gamma ; H, \nu\rangle$ is a quasi-colored bounded lattice, then $\langle H ; \nu\rangle$ is a quasiordered set with a least element and a greatest element; possibly with many least elements and many greatest elements. For $\langle x, y\rangle \in L, \gamma(\langle x, y\rangle)$ is called the color (rather than the quasi-color) of $\langle x, y\rangle$.

4.2. Two technical lemmas. Recently, G. Grätzer has proved the following two statements. They will be very useful in this paper.

Lemma 4.1 (G. Grätzer [13]). Let $L$ be a lattice such that every interval of $L$ is of finite length. Let $\delta$ be an equivalence relation on $L$ with intervals as equivalence classes. Then $\delta$ is a congruence relation iff the following condition and its dual hold for every $x, y, z \in L$ :

$$
\text { If } x \prec y, x \prec z \text { and }\langle x, y\rangle \in \delta \text {, then }\langle z, y \vee z\rangle \in \delta \text {. }
$$

For $i \in\{1,2\}$, let $\mathfrak{p}_{i}=\left[x_{i}, y_{i}\right]$ be prime intervals of a lattice $L$. That is, $\left\langle x_{i}, y_{i}\right\rangle \in \operatorname{Pairs}^{\prec}(L)$. We say that $\mathfrak{p}_{1}$ is prime-perspective down to $\mathfrak{p}_{2}$, denoted by $\mathfrak{p}_{1} \stackrel{\text { p-dn }}{\rightarrow} \mathfrak{p}_{2}$ or $\left\langle x_{1}, y_{1}\right\rangle \stackrel{\text { p-dn }}{\rightarrow}\left\langle x_{2}, y_{2}\right\rangle$, if $y_{1}=x_{1} \vee y_{2}$ and $x_{1} \wedge y_{2} \leq x_{2}$; see Figure 5, where the solid lines indicate prime intervals while the dotted ones stand for the ordering relation of $L$. We define prime-perspective up, denoted by $\mathfrak{p}_{1} \stackrel{\text { p-up }}{\longrightarrow} \mathfrak{p}_{2}$ or $\left\langle x_{1}, y_{1}\right\rangle \stackrel{\text { p-up }}{\longrightarrow}\left\langle x_{2}, y_{2}\right\rangle$, dually. We say that $\mathfrak{p}_{1}$ is prime-perspective to $\mathfrak{p}_{2}$, in notation, $\mathfrak{p}_{1} \stackrel{\text { p-pr }}{\longrightarrow} \mathfrak{p}_{2}$, if $\mathfrak{p}_{1} \stackrel{\text { p-dn }}{\rightarrow} \mathfrak{p}_{2}$ or $\mathfrak{p}_{1} \stackrel{\text { p-up }}{\longrightarrow} \mathfrak{p}_{2}$.
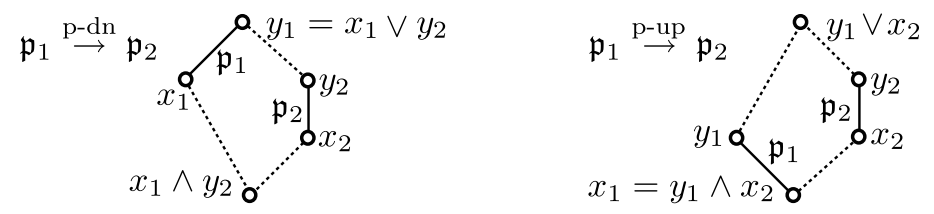

FiguRE 5. Prime perspectivities

Lemma 4.2 (Prime-Projectivity Lemma; see G. Grätzer [14]). Let $L$ be a lattice of finite length. Assume that $\left[u_{1}, v_{1}\right]$ and $\left[u_{2}, v_{2}\right]$ are prime intervals in $L$, that is, $\left\langle u_{1}, v_{1}\right\rangle,\left\langle u_{2}, v_{2}\right\rangle \in \operatorname{Pairs}^{\prec}(L)$ are covering pairs. Then $\operatorname{con}\left(u_{1}, v_{1}\right) \leq \operatorname{con}\left(u_{2}, v_{2}\right)$ iff there exist a nonnegative integer $n$ and a sequence $\left\langle x_{0}, y_{0}\right\rangle,\left\langle x_{1}, y_{1}\right\rangle, \ldots,\left\langle x_{n}, y_{n}\right\rangle$ of covering pairs such that $\left\langle x_{0}, y_{0}\right\rangle=\left\langle u_{2}, v_{2}\right\rangle$, $\left\langle x_{n}, y_{n}\right\rangle=\left\langle u_{1}, v_{1}\right\rangle$, and $\left\langle x_{i-1}, y_{i-1}\right\rangle \stackrel{\mathrm{p}-\mathrm{pr}}{\rightarrow}\left\langle x_{i}, y_{i}\right\rangle$ for all $i \in\{1, \ldots, n\}$.

4.3. Basic gadgets. For parameters $p \neq q$, the quasi-colored lattice

$$
\mathcal{G}^{\mathrm{up}}(p, q)=\left\langle G^{\mathrm{up}}(p, q), \lambda_{p q}^{\mathrm{up}} ; \gamma_{p q}^{\mathrm{up}} ; H(p, q), \nu_{p q}\right\rangle
$$

depicted in Figure 6 is our upward gadget. (Its "lattice part" is a lattice by, say, D. Kelly and I. Rival [26, Corollary 2.4].) The upward gadget consists of a 17-element lattice $G^{\text {up }}(p, q)=\left\langle G^{\text {up }}(p, q) ; \leq\right\rangle=\left\langle G^{\text {up }}(p, q) ; \lambda_{p q}^{\text {up }}\right\rangle$, a 4-element 

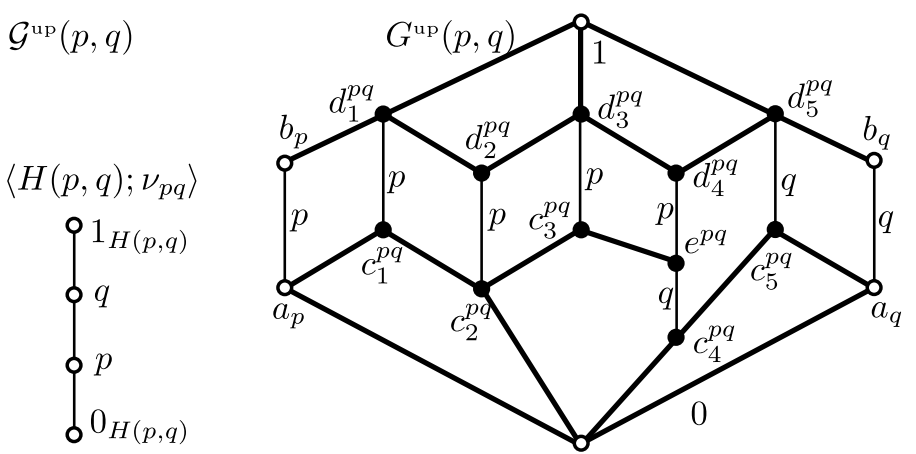

FIGURE 6 . The (upward) gadget, $\mathcal{G}^{\mathrm{up}}(p, q)$

quasiordered set $\left\langle H(p, q) ; \nu_{p q}\right\rangle$, which is actually a chain, and the quasi-coloring $\gamma_{p q}^{\mathrm{up}}$ is defined by the figure as follows: for $\langle x, y\rangle \in \operatorname{Pairs}^{\leq}\left(G^{\mathrm{up}}(p, q)\right)$,

$$
\gamma_{p q}^{\mathrm{up}}(\langle x, y\rangle)= \begin{cases}p, & \text { if }\langle x, y\rangle \text { is a } p \text {-colored edge in the figure, } \\ q, & \text { if }\langle x, y\rangle \text { is a } q \text {-colored edge, } \\ q, & \text { if }\langle x, y\rangle=\left\langle c_{4}^{p q}, d_{4}^{p q}\right\rangle, \\ 0_{H(p, q)}, & \text { if } x=y, \\ 1_{H(p, q)}, & \text { otherwise (if }[x, y] \text { contains a thick edge). }\end{cases}
$$

The adjective "upward" comes from the fact that in order to get from $a_{p}$ to $c_{1}^{p q}$, or from $b_{p}$ to $d_{1}^{p q}$, we have to go upwards; see Figure 6. Using Lemma 4.2, it is straightforward to see that $\mathcal{G}^{\text {up }}(p, q)$ is a quasi-colored lattice.

Remark 4.3. G. Grätzer [12] uses a different technique and his gadget, denoted by $S=S(p, q)$ in [12], cannot be quasi-colored by a four element chain. Also, while (4.7) will turn our $G^{\text {up }}(p, q)$ into a selfdual lattice, the analogous construction with his $S(p, q)$ would not give a lattice. These are the reasons that we need a larger gadget; however, the size $\left|G^{\text {up }}(p, q)\right|=17$ seems to be optimal for our purpose.

We obtain the downward gadget lattice

$$
\mathcal{G}^{\mathrm{dn}}(p, q)=\left\langle G^{\mathrm{dn}}(p, q), \lambda_{p q}^{\mathrm{dn}} ; \gamma_{p, q}^{\mathrm{dn}} ; H(p, q), \nu_{p q}\right\rangle
$$

by taking the dual

$$
\left\langle G^{\mathrm{dn}}(p, q) ; \lambda_{p q}^{\mathrm{dn}}\right\rangle:=\left\langle G^{\mathrm{up}}(p, q) ;\left(\lambda_{p q}^{\mathrm{up}}\right)^{-1}\right\rangle
$$

of the lattice $\left\langle G^{\mathrm{up}}(p, q) ; \lambda_{p q}^{\mathrm{up}}\right\rangle$ and defining $\gamma_{p q}^{\mathrm{dn}}$ by the rule

$$
\gamma_{p q}^{\mathrm{dn}}(\langle x, y\rangle):=\gamma_{p q}^{\mathrm{up}}(\langle y, x\rangle) \quad \text { for }\langle x, y\rangle \in \text { Pairs }^{\leq}\left(G^{\mathrm{dn}}(p, q)\right),
$$

that is, for $\langle y, x\rangle \in$ Pairs $^{\leq}\left(G^{\text {up }}(p, q)\right)$; see Figure 7 . The upward gadget and the downward one are our basic gadgets. 

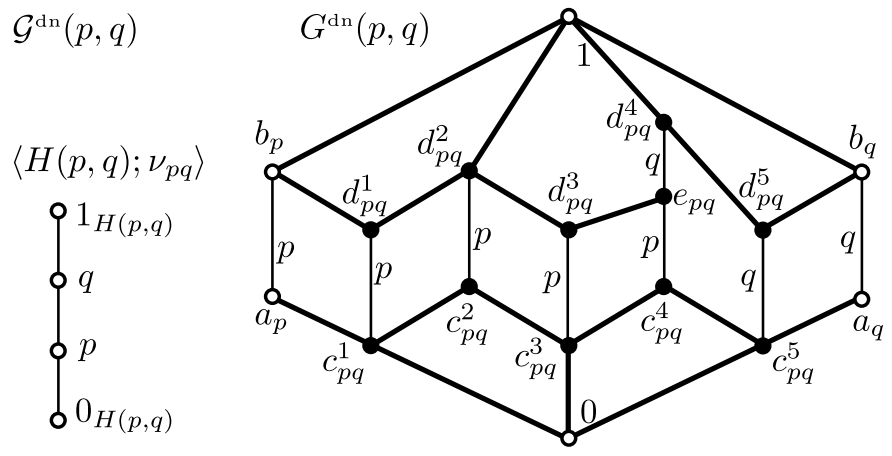

FiguRE 7 . The downward gadget, $\mathcal{G}^{\mathrm{dn}}(p, q)$

If $[x, y]$ and $\left[x^{\prime}, y^{\prime}\right]$ are intervals of a lattice such that $\left\{x, y, x^{\prime}, y^{\prime}\right\}$ is a nonchain sublattice, then $[x, y]$ and $\left[x^{\prime}, y^{\prime}\right]$ are transposed or, in other words, perspective intervals, and $\langle x, y\rangle$ and $\left\langle x^{\prime}, y^{\prime}\right\rangle$ are perspective ordered pairs. The following convention applies to all of our figures that contain both thin and thick edges: if $\gamma$ is a quasi-coloring, then for an ordered pair $\langle x, y\rangle$,

$$
\gamma(\langle x, y\rangle)= \begin{cases}0, & \text { iff } x=y, \\ u, & \text { if } x \prec y \text { is a thin edge labeled by } u, \\ 1, & \text { if the interval }[x, y] \text { contains is a thick edge, } \\ \gamma\left(\left\langle x^{\prime}, y^{\prime}\right\rangle\right), & \text { if }[x, y] \text { and }\left[x^{\prime}, y^{\prime}\right] \text { are transposed intervals. }\end{cases}
$$

By this convention and the following lemma, our figures with thin and thick edges determine the corresponding quasi-colorings. In order to formulate this lemma, let $\langle H ; \nu\rangle$ be a quasiordered set. For $p, q_{1}, \ldots, q_{n} \in H$, we say that $p \in H$ is a join of the elements $q_{1}, \ldots, q_{n} \in H$ if $q_{i} \leq_{\nu} p$ for all $i$ and, for every $r \in H$, the conjunction of $q_{i} \leq_{\nu} r$ for $i=1, \ldots, n$ implies $p \leq_{\nu} r$. Even if a join exists, it need not be unique in the usual sense, but it is unique modulo $\Theta_{\nu}=\nu \cap \nu^{-1}$. The easy statement below is taken from G. Czédli $[5$, Lemma 4.6].

Lemma 4.4. If $u_{0} \leq u_{1} \leq \cdots \leq u_{n}$ are elements of a quasi-colored lattice $\langle L, \leq ; \gamma ; H, \nu\rangle$, then

$$
\gamma\left(\left\langle u_{0}, u_{n}\right\rangle\right)={ }_{\nu} \bigvee_{i=1}^{n} \gamma\left(\left\langle u_{i-1}, u_{i}\right\rangle\right) \quad \text { holds in }\langle H ; \nu\rangle .
$$

Although $G^{\mathrm{dn}}(p, q)$ and $G^{\mathrm{dn}}(u, v)$ are isomorphic in a self-explanatory sense, we do not consider them equal if $\langle p, q\rangle \neq\langle u, v\rangle$. Actually, we always assume that, for $\langle p, q\rangle \neq\langle u, v\rangle$,

the intersection of any two of $G^{\mathrm{up}}(p, q), G^{\mathrm{up}}(u, v), G^{\mathrm{dn}}(p, q)$, and $G^{\mathrm{dn}}(u, v)$ is as small as it follows from the notation.

For example, if $|\{p, q, u\}|=3$, then $G^{\mathrm{up}}(p, q) \cap G^{\mathrm{dn}}(p, u)=\left\{0, a_{p}, b_{p}, 1\right\}$ and $G^{\mathrm{up}}(p, q) \cap G^{\mathrm{up}}(q, p)=G^{\mathrm{up}}(p, q) \cap G^{\mathrm{dn}}(p, q)=\left\{0, a_{p}, b_{p}, a_{q}, b_{q}, 1\right\}$. 
4.4. More about gadgets. Convention (4.6) allows us to speak of unions easily, and these unions are bounded ordered sets. For example, we need the ordered set

$$
G^{\mathrm{db}}(p, q):=G^{\mathrm{up}}(p, q) \cup G^{\mathrm{dn}}(p, q) ;
$$

which is the lattice from Figure 4; the ordering is understood in the natural way. Although it would not be hard to verify that $G^{\mathrm{db}}(p, q)$ is a lattice, we conclude this fact from the following lemma, which will also be needed later.

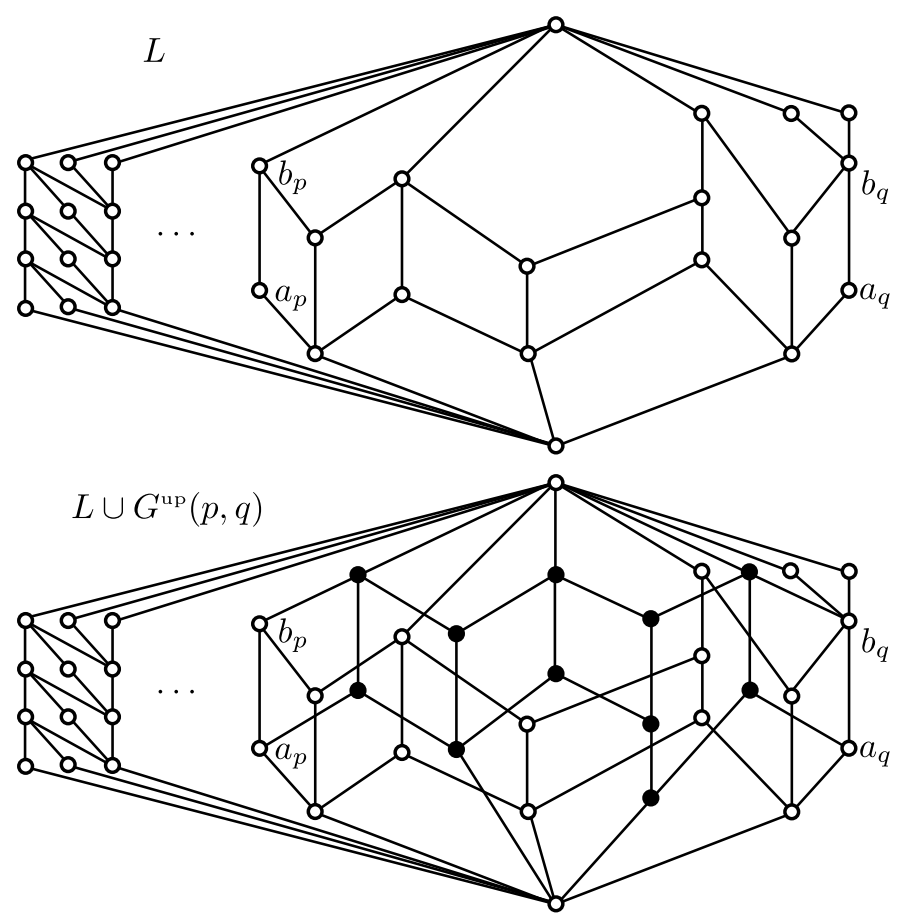

Figure 8. G. Inserting the upward gadget $G^{\text {up }}(p, q)$

Lemma 4.5. Assume that $L=\left\langle L ; \leq_{L}\right\rangle=\left\langle L ; \lambda_{L}\right\rangle$ is a lattice of length 5 , and let $0<a_{p} \prec b_{p}<1$ and $0<a_{q} \prec b_{q}<1$ in $L$ such that none of the intervals $\left[0, b_{p}\right],\left[a_{p}, 1\right],\left[0, b_{q}\right]$, and $\left[a_{q}, 1\right]$ is of length greater than 3 . Assume that $a_{p} \vee a_{q}=1, b_{p} \wedge b_{q}=0$, and $L \cap G^{\mathrm{up}}(p, q)=\left\{0, a_{p}, b_{p}, a_{q}, b_{q}, 1\right\}$. Let

$$
L^{\Delta}:=L \cup G^{\text {up }}(p, q) \text { and } \lambda^{\Delta}:=\operatorname{quo}\left(\lambda_{L} \cup \lambda_{p q}^{\text {up }}\right) \text {; }
$$

see Figure 8. Then $L^{\Delta}=\left\langle L^{\Delta} ; \lambda^{\Delta}\right\rangle$, also denoted by $L_{p, q}^{\Delta}$ or $\left\langle L_{p, q}^{\Delta} ; \leq^{\Delta}\right\rangle$, is a lattice of length 5 . Furthermore, both $L$ and $G^{\mathrm{up}}(p, q)$ are $\{0,1\}$-sublattices of $L^{\Delta}$.

We say that $L^{\Delta}$ is obtained from $L$ by inserting an upward gadget. For an ordered set $P$ and $\varnothing \neq X \subseteq P$, the least order ideal including $X$ is denoted by $\downarrow_{P} X$ or, if $P$ is understood, by $\downarrow X$. For $x \in P$, we write $\downarrow x$ rather than $\downarrow\{x\}$. The order filter $\uparrow_{P} x$ is defined dually. 
Proof of Lemma 4.5. For brevity, we will often write $G^{\text {up }}, \uparrow_{G} x$, and $\leq_{G}$ instead of $G^{\mathrm{up}}(p, q), \uparrow_{G_{\mathrm{p}}^{\mathrm{up}}(p, q)} x$, and $\lambda_{p q}^{\mathrm{up}}$, respectively. Let

$$
B=B(p, q):=\left\{0, a_{p}, b_{p}, a_{q}, b_{q}, 1\right\}=L \cap G^{\text {up }}(p, q) .
$$

Since $B$ is a complete $\{0,1\}$-sublattice of both $L$ and $G^{\mathrm{up}}(p, q)$, we can consider the following closure operators

${ }^{*}: G^{\text {up }} \rightarrow B$, where $x^{*}$ is the smallest element of $B \cap \uparrow_{G} x$,

$\bullet: L \rightarrow B$, where $x^{\bullet}$ is the smallest element of $B \cap \uparrow_{L} x$

and, dually, the interior operators

${ }_{*}: G^{\text {up }} \rightarrow B$, where $x_{*}$ is the largest element of $B \cap \downarrow_{G} x$,

- : $L \rightarrow B$, where $x_{\bullet}$ is the largest element of $B \cap \downarrow_{L} x$.

For a subset $X$ of $Y$ and a relation $\varrho \subseteq Y^{2}$, the restriction $\varrho \cap X^{2}$ of $\varrho$ to $X$ is denoted by $\varrho\rceil_{X}$. We claim that

$\lambda^{\Delta}$ is an ordering, $\left.\left.\quad \lambda^{\Delta}\right\rceil_{L}=\lambda_{L}, \quad \lambda^{\Delta}\right\rceil_{G^{\text {up }}}=\lambda_{p q}^{\text {up }}$,

for $x \in L$ and $y \in G^{\text {up }}, x \leq^{\Delta} y \Longleftrightarrow x \leq_{G} y \Longleftrightarrow x \leq_{L} y_{*}$,

for $x \in G^{\text {up }}$ and $y \in L, x \leq^{\Delta} y \Longleftrightarrow x^{*} \leq_{L} y \Longleftrightarrow x \leq_{G} y_{\bullet}$.

In order to verify this, observe that the second " $\Longleftrightarrow$ " holds in the last two lines of (4.11). Hence, we can define a new relation $\lambda^{\prime}$ by (4.11) with $\lambda^{\prime}$ in place of $\lambda^{\Delta}$ and $\leq^{\Delta}$. It is straightforward to verify that $\lambda^{\prime}$ is a quasiordering; a part of the argument for antisymmetry runs as follows. Let, say, $x \in L$ and $y \in G^{\text {up }}$ such that $\langle x, y\rangle,\langle y, x\rangle \in \lambda^{\prime}$. Then $x \leq_{L} x^{\bullet} \leq_{G} y \leq_{G} y^{*} \leq_{L} x$. Since $x^{\bullet} \leq_{G} y^{*}$ and these elements are in $B$, we have that $x \leq_{L} x^{\bullet} \leq_{L} y^{*} \leq_{L} x$. Using antisymmetry in $L$, we obtain that $x=x^{\bullet}=y^{*}$. Combining this with $x^{\bullet} \leq_{G} y \leq_{G} y^{*}$, we obtain that $x=y$, as required. Finally, armed with the fact that $\lambda^{\prime}$ is a quasiordering, we obtain that $\lambda^{\Delta}=\lambda^{\prime}$, proving (4.11).

Note that $x^{*}=1$ for all $x \in G^{\text {up }} \backslash L$. Thus, $\uparrow_{L^{\Delta}}\left(G^{\text {up }} \backslash L\right)=\left(G^{\text {up }} \backslash L\right) \cup\{1\}$, which is the second reason that $G^{\text {up }}$ is called an upward gadget.

Next, in order to show that $L^{\Delta}$ is a lattice, let $x, y \in L^{\Delta}$. We need to prove the existence of $x \vee^{\Delta} y:=x \vee_{L^{\Delta}} y$ and $x \wedge^{\Delta} y:=x \wedge_{L} \Delta y$. Denoting the lattice operations in $L$ and $G^{\text {up }}$ by $\vee_{L}, \wedge_{L}$, and $\vee_{G}, \wedge_{G}$, respectively, we claim that

$$
\begin{aligned}
& \text { if } x \in L \backslash G^{\text {up }} \text { and } y \in G^{\text {up }} \backslash L, \text { then } x \wedge^{\Delta} y=x \wedge_{L} y_{*}, \\
& \text { if } x \in L \backslash G^{\text {up }} \text { and } y \in G^{\text {up }} \backslash L \text {, then } x \vee^{\Delta} y=x \vee_{G} y, \\
& \text { if } x, y \in L \text {, then } x \wedge^{\Delta} y=x \wedge_{L} y \text {, and } x \vee^{\Delta} y=x \vee_{L} y, \\
& \text { if } x, y \in G^{\text {up }} \text {, then } x \wedge^{\Delta} y=x \wedge_{G} y \text {, and } x \vee^{\Delta} y=x \vee_{G} y \text {. }
\end{aligned}
$$

We can assume that $\{x, y\} \cap\{0,1\}=\varnothing$. Since $\left(G^{\text {up }} \backslash L\right) \cap \downarrow_{L^{\Delta}} x=\varnothing$ for $x \in L \backslash G^{\text {up }},(4.12)$ is clear. Similarly, $\left(L \backslash G^{\text {up }}\right) \cap \uparrow_{L^{\Delta}} y=\varnothing$ for $y \in G^{\text {up }} \backslash L$, and we obtain (4.13). Next, let $x, y \in L$, and let $z \in G^{\text {up }}$ be a lower bound of $\{x, y\}$ in $L^{\Delta}$. By (4.11), $z^{*} \leq_{L} x$ and $z^{*} \leq_{L} y$, so $z^{*} \leq_{L} x \wedge_{L} y$. Using (4.11) again, $z \leq^{\Delta} x \wedge_{L} y$. This gives the first equality in (4.14). In order to show the second one, let $u \in G^{\text {up }}$ be an upper bound of $x$ and $y$. (4.11) gives that $x \leq_{L} u_{*}$ and 
$y \leq_{L} u_{*}$, and we obtain that $x \vee_{L} y \leq_{L} u_{*} \leq_{G} u$. Hence, $x \vee_{L} y \leq^{\Delta} u$, proving the second equality in (4.14). Since (4.15) follows analogously, $L^{\Delta}$ is a lattice. By (3.3) and the assumption on lengths in the lemma, $L^{\Delta}$ is of length 5 .

It follows from Lemma 4.5 that $G^{\mathrm{db}}(p, q)$, see (4.7) and Figure 4, is a lattice. It is a selfdual lattice of length 5 . The ordering on this lattice, denoted by $\lambda_{p q}^{\mathrm{db}}$, is the quasiorder generated by $\lambda_{p q}^{\mathrm{up}} \cup \lambda_{p q}^{\mathrm{dn}}$. Since $\gamma_{p q}^{\mathrm{up}}$ and $\gamma_{p q}^{\mathrm{dn}}$ are not in conflict on Pairs $\leq\left(G^{\text {up }}\right) \cap$ Pairs $\leq\left(G^{\mathrm{dn}}\right)=\lambda_{p q}^{\text {up }} \cap \lambda_{p q}^{\mathrm{dn}}$, we have a map

$$
\gamma_{p q}^{\mathrm{up}} \cup \gamma_{p q}^{\mathrm{dn}}: \text { Pairs } \leq\left(G^{\mathrm{up}}\right) \cup \text { Pairs }^{\leq}\left(G^{\mathrm{dn}}\right) \rightarrow H(p, q) .
$$

Letting $\gamma_{p q}^{\mathrm{db}}(\langle x, y\rangle)=1_{H(p, q)}$ for all pair $\langle x, y\rangle \in$ Pairs $\leq\left(G^{\mathrm{ab}}\right)$ not belonging to Pairs $\leq\left(G^{\mathrm{up}}\right) \cup$ Pairs $\leq\left(G^{\mathrm{dn}}\right)$, we obtain a well-defined extension $\gamma_{p q}^{\mathrm{db}}$ of $\gamma_{p q}^{\mathrm{up}} \cup \gamma_{p q}^{\mathrm{dn}}$ to Pairs $\leq\left(G^{\mathrm{db}}\right)$. Equivalently, $\gamma_{p q}^{\mathrm{db}}$ : Pairs ${ }^{\leq}\left(G^{\mathrm{db}}\right) \rightarrow H(p, q)$ is determined by Figure 4, convention (4.4), and Lemma 4.4. Using Lemmas 4.1 and 4.2, it follows in a straightforward way that $\gamma_{p q}^{\mathrm{db}}$ is a quasi-coloring. So we obtain a quasi-colored lattice

$$
\mathcal{G}^{\mathrm{db}}(p, q)=\left\langle G^{\mathrm{db}}(p, q), \lambda_{p q}^{\mathrm{db}} ; \gamma_{p q}^{\mathrm{db}} ; H(p, q), \nu_{p q}^{\mathrm{db}}\right\rangle,
$$

which we call the double gadget.

We define a polarity $\pi$ on $G^{\mathrm{db}}(p, q)$ as Figures 4,6 , and 7 suggest. In particular, $\pi\left(a_{p}\right)=b_{p}, \pi\left(a_{q}\right)=b_{q}, \pi\left(e^{p q}\right)=e_{p q}, \pi\left(c_{i}^{p q}\right)=d_{p q}^{i}$, and $\pi\left(d_{i}^{p q}\right)=$ $c_{p q}^{i}$, for $i \in\{1, \ldots, 5\}$. It is straightforward to conclude from (C1), (C2), (4.3), and (4.4) that $\left\langle G^{\mathrm{db}}(p, q), \lambda_{p q}^{\mathrm{db}}, \pi\right\rangle \in \mathbf{P L a t}_{(2.4)}^{\mathrm{emb}}$.

4.5. Constructing large quasi-colored lattices. Let $H$ be an arbitrary set such that $0 \in H, 1 \in H$ and $0 \neq 1$. As in (3.1), $H^{-01}$ stands for $H \backslash\{0,1\}$. The selfdual simple lattice depicted twice in Figure 9 is denoted by $M_{4 \times 3}$. Its polarity is the rotational symmetry on the left of the figure. Note that, instead of $M_{4 \times 3}$, we could use any selfdual lattice $M$ satisfying (3.3); the role of length $(M)=5$ is to guarantee that $L(H, \varnothing, \varnothing)$ in Figure 9 and, thus, $L(H, I, J)$ later in (4.19) are of length 5 rather than of length at most 5 . Note also that $a_{0}=b_{0}$ is an arbitrarily fixed element of $M_{4 \times 3}$ (in a non-crowded part of Figure 9). For each $p \in H^{-01}$, take a 4-element chain $C_{p}:=\left\{0 \prec a_{p} \prec\right.$ $\left.b_{p} \prec 1\right\}$. The ordering on this chain and that of the lattice $M_{4 \times 3}$ will also be denoted by $\lambda_{C_{p}}$ and $\lambda_{M_{4 \times 3}}$, respectively. We assume that $H, M_{4 \times 3}$ and all the $C_{p}$ are as much disjoint as the notation allows, that is, the intersection of any two is $\{0,1\}$. Writing $\bigcup_{p}$ for $\bigcup_{p \in H^{-01}}$, let

$$
\left\langle L(H, \varnothing, \varnothing) ; \lambda_{H, \varnothing, \varnothing}\right\rangle:=\left\langle M_{4 \times 3} \cup \bigcup_{p} C_{p} ; \lambda_{M_{4 \times 3}} \cup \bigcup_{p} \lambda_{C_{p}}\right\rangle,
$$

which is a obviously a lattice; see on the right of Figure 9. Its polarity extends that of $M_{4 \times 3}$ with the reflection across a horizontal axis. The polarity preserves the quasi-coloring, which is indicated in the figure according to (4.4). Hence, by $(\mathrm{C} 1)$ and $(\mathrm{C} 2), L(H, \varnothing, \varnothing)$ with its polarity becomes a member of $\mathbf{P L a t}_{(2.4)}^{\mathrm{emb}}$. 

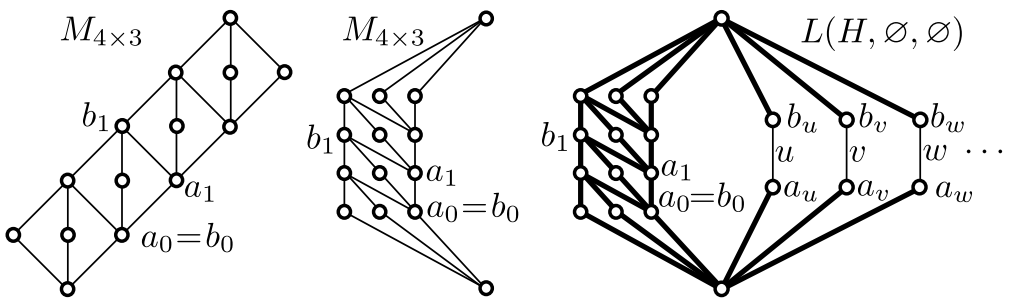

Figure 9. $M_{4 \times 3}$ and $L(H, \varnothing, \varnothing)$ for $H=\{0,1, u, v, w, \ldots\}$

Next, we insert several upward gadgets and, dually, downward gadgets into $L(H, \varnothing, \varnothing)$; see the paragraph after Lemma 4.5. With $H$ and $L(H, \varnothing, \varnothing)$ as above, let us agree that, for every $p \neq q \in H \backslash\{0\}$,

$$
G^{\mathrm{up}}(p, q) \cap L(H, \varnothing, \varnothing)=G^{\mathrm{dn}}(p, q) \cap L(H, \varnothing, \varnothing)=\left\{0, a_{p}, b_{p}, a_{q}, b_{q}, 1\right\} .
$$

Assume that

$$
\begin{aligned}
& I \text { and } J \text { are subsets of }(H \backslash\{0\}) \times(H \backslash\{0\}) \\
& \text { such that } p \neq q \text { holds for every }\langle p, q\rangle \in I \cup J .
\end{aligned}
$$

Taking Conventions (4.6) and (4.17) into account, we define

$$
\begin{aligned}
L(H, I, J) & :=L(H, \varnothing, \varnothing) \cup \bigcup_{\langle p, q\rangle \in I} G^{\mathrm{up}}(p, q) \cup \bigcup_{\langle p, q\rangle \in J} G^{\mathrm{dn}}(p, q), \text { and } \\
\lambda_{H, I, J} & :=\operatorname{quo}\left(\lambda_{H, \varnothing, \varnothing} \cup \bigcup_{\langle p, q\rangle \in I} \lambda_{p q}^{\mathrm{up}} \cup \bigcup_{\langle p, q\rangle \in J} \lambda_{p q}^{\mathrm{dn}}\right) .
\end{aligned}
$$

As opposed to (4.16), the mere union in the second line of (4.19) is not sufficient to obtain a quasiordering. Observe that, for $\langle p, q\rangle \in I$ and $I^{\prime}:=I \backslash\{\langle p, q\rangle\}$,

$\left\langle L(H, I, J) ; \lambda_{H, I, J}\right\rangle$ is obtained from $\left\langle L\left(H, I^{\prime}, J\right) ; \lambda_{H, I^{\prime}, J}\right\rangle$ by inserting the upward gadget $G^{\mathrm{up}}(p, q)$ at $\left\{0, a_{p}, b_{p}, a_{q}, b_{q}, 1\right\}$,

and analogously with $J$ and "downward" instead of $I$ and "upward". Hence, a straightforward transfinite induction based on Lemma 4.5 yields that

$$
\left\langle L(H, I, J) ; \lambda_{H, I, J}\right\rangle \text { is a lattice of length } 5
$$

and, furthermore, if $H_{1} \subseteq H_{2}, I_{1} \subseteq I_{2}$, and $J_{1} \subseteq J_{2}$, then

$$
\left\langle L\left(H_{1}, I_{1}, J_{1}\right) ; \lambda_{H_{1}, I_{1}, J_{1}}\right\rangle \text { is a sublattice of }\left\langle L\left(H_{2}, I_{2}, J_{2}\right) ; \lambda_{H_{2}, I_{2}, J_{2}}\right\rangle \text {. }
$$

Next, we turn the lattice $\left\langle L(H, I, J) ; \lambda_{H, I, J}\right\rangle$ into a quasi-colored lattice. Let $\nu_{H, \varnothing, \varnothing}$ be the unique ordering of $H$, with least element 0 and largest element 1 , such that $\left\langle H ; \leq_{H, \varnothing, \varnothing\rangle}\right\rangle:=\left\langle H ; \nu_{H, \varnothing, \varnothing}\right\rangle$ is a modular lattice of length 2. That is, denoting the covering relation with respect to $\nu_{H, \varnothing, \varnothing}$ by $\prec_{H, \varnothing, \varnothing}$,

$0 \prec_{H, \varnothing, \varnothing} p \prec_{H, \varnothing, \varnothing} 1$ for all $p \in H^{-01}$, and any $p \neq q \in H^{-01}$ are incomparable with respect to $\nu_{H, \varnothing, \varnothing}$. 
In accordance with Figure 9 and (4.4), for $\langle x, y\rangle \in$ Pairs $^{\leq}(L(H, \varnothing, \varnothing))$, we let

$$
\gamma_{H, \varnothing, \varnothing}(\langle x, y\rangle)= \begin{cases}p, & \text { if }\langle x, y\rangle=\left\langle a_{p}, b_{p}\right\rangle \text { and } p \in H \backslash\{0\}, \\ 0, & \text { if } x=y, \\ 1, & \text { otherwise. }\end{cases}
$$

It is straightforward to see that $\left\langle L(H, \varnothing, \varnothing), \lambda_{H, \varnothing, \varnothing} ; \gamma_{H, \varnothing, \varnothing} ; H, \nu_{H, \varnothing, \varnothing}\right\rangle$ is a quasi-colored lattice. The quasi-colorings $\gamma_{p q}^{\mathrm{up}}$ and $\gamma_{p q}^{\mathrm{dn}}$ for $p \neq q \in H^{-01}$ are not in conflict with $\gamma_{H, \varnothing, \varnothing}$. Furthermore, although the maps $\gamma_{p 1}^{\text {up }}, \gamma_{1 p}^{\text {up }}$, $\gamma_{p 1}^{\mathrm{dn}}$ and $\gamma_{1 p}^{\mathrm{dn}}$, defined by (4.2) and (4.3), are not quasi-colorings, these maps are not in conflict with $\gamma_{H, \varnothing, \varnothing}$ either. Therefore, there is a unique map $\gamma_{H, I, J}:$ Pairs $\leq(L(H, I, J)) \rightarrow H$ such that

$$
\gamma_{H, I, J}(\langle x, y\rangle)= \begin{cases}\gamma_{H, \varnothing, \varnothing}(\langle x, y\rangle), & \text { if }\langle x, y\rangle \in \text { Pairs } \leq(L(H, \varnothing, \varnothing)), \\ \gamma_{p q}^{\mathrm{up}}(\langle x, y\rangle), & \text { if }\langle x, y\rangle \in \text { Pairs }^{\mathrm{u}} \leq\left(G^{\mathrm{up}}(p, q)\right), \\ \gamma_{p q}^{\mathrm{dn}}(\langle x, y\rangle), & \text { if }\langle x, y\rangle \in \text { Pairs }^{\mathrm{an}} \leq\left(G^{\mathrm{dn}}(p, q)\right), \\ 1, & \text { otherwise. }\end{cases}
$$

Finally, after letting

$$
\nu_{H, I, J}:=\mathrm{quo}_{H}\left(\nu_{H, \varnothing, \varnothing} \cup I \cup J\right),
$$

we are ready to formulate the key lemma of this section. Its importance will be shown later by Lemma 4.7 .

Lemma 4.6. Assume (4.18). Then

$$
\mathcal{L}(H, I, J):=\left\langle L(H, I, J), \lambda_{H, I, J} ; \gamma_{H, I, J} ; H, \nu_{H, I, J}\right\rangle
$$

is a quasi-colored lattice of length 5 . If $I=J$, then it is a selfdual lattice.

Proof. We know from (4.21) that $\left\langle L(H, I, J), \lambda_{H, I, J}\right\rangle$ is a lattice. As usual, projectivity is the reflexive transitive closure of the relation "perspectivity". It follows from the construction, see Figures 6 and 7, that, for every $\langle x, y\rangle \in$ Pairs $\leq(L(H, I, J))$,

$$
\text { if } \gamma_{H, I, J}(\langle x, y\rangle)=p \in H^{-01} \text {, then }\langle x, y\rangle \text { is projective to }\left\langle a_{p}, b_{p}\right\rangle \text {. }
$$

The largest and the smallest congruence of a bounded lattice $K$ will be denoted by $\nabla_{K}$ and $\Delta_{K}$, respectively. They belong to Princ $(K)$, because $\nabla_{K}=$ $\operatorname{con}_{K}(0,1)$. Using that $\gamma_{H, \varnothing, \varnothing}$ and the $\gamma_{p q}^{\text {up }}$ and $\gamma_{p q}^{\mathrm{dn}}$ are quasi-colorings and so they satisfy $(\mathrm{C} 1)$, we conclude that for every $\langle x, y\rangle \in$ Pairs $^{\leq}(L(H, I, J))$,

$$
\text { if } \gamma_{H, I, J}(\langle x, y\rangle)=1 \text {, then } \operatorname{con}(x, y)=\nabla_{L(H, I, J)} \text {. }
$$

In order to prove that $\mathcal{L}(H, I, J)$ satisfies $(\mathrm{C} 1)$, assume that $\left\langle x_{1}, y_{1}\right\rangle$ and $\left\langle x_{2}, y_{2}\right\rangle$ belong to Pairs ${ }^{\leq}(L(H, I, J)), p=\gamma_{H, I, J}\left(\left\langle x_{1}, y_{1}\right\rangle\right), q=\gamma_{H, I, J}\left(\left\langle x_{2}, y_{2}\right\rangle\right)$, and $\langle p, q\rangle \in \nu_{H, I, J}$. We need to show that $\operatorname{con}\left(x_{1}, y_{1}\right) \leq \operatorname{con}\left(x_{2}, y_{2}\right)$. This is trivial if $p=q$ or $p=0$. It is also trivial by (4.27) if $q=1$. Hence, we assume that $\{p, q\} \cap\{0,1\}=\varnothing$. Based on (4.24), it suffices to deal only with the case $\langle p, q\rangle \in \nu_{H, \varnothing, \varnothing} \cup I \cup J$. However, $\langle p, q\rangle \in \nu_{H, \varnothing, \varnothing}$ has already been 
excluded, because $p \neq q$ and $\{p, q\} \cap\{0,1\}=\varnothing$. Thus, by duality, we can assume that $\langle p, q\rangle \in I$. Since $\left\langle x_{1}, y_{1}\right\rangle$ is projective to $\left\langle a_{p}, b_{p}\right\rangle$ by (4.26) and since projective pairs generate the same congruence, $\operatorname{con}\left(x_{1}, y_{1}\right)=\operatorname{con}\left(a_{p}, b_{p}\right)$. Similarly, $\operatorname{con}\left(x_{2}, y_{2}\right)=\operatorname{con}\left(a_{q}, b_{q}\right)$. Since $\langle p, q\rangle \in I, G^{\mathrm{up}}(p, q)$ is a sublattice of $L(H, I, J)$ and $a_{p}, b_{p}, a_{q}$, and $b_{q}$ belong to this sublattice. Therefore, as Figure 6 shows,

$$
\begin{aligned}
\left\langle a_{q}, b_{q}\right\rangle & \stackrel{\text { p-up }}{\rightarrow}\left\langle c_{5}^{p q}, d_{5}^{p q}\right\rangle \stackrel{\text { p-dn }}{\rightarrow}\left\langle e^{p q}, d_{4}^{p q}\right\rangle \stackrel{\text { p-up }}{\rightarrow}\left\langle c_{3}^{p q}, d_{3}^{p q}\right\rangle \\
& \stackrel{\text { p-dn }}{\rightarrow}\left\langle c_{2}^{p q}, d_{2}^{p q}\right\rangle \stackrel{\text { p-up }}{\rightarrow}\left\langle c_{1}^{p q}, d_{1}^{p q}\right\rangle \stackrel{\text { p-dn }}{\rightarrow}\left\langle a_{p}, b_{p}\right\rangle .
\end{aligned}
$$

Hence, by (the trivial direction of) Lemma $4.2, \operatorname{con}\left(a_{p}, b_{p}\right) \leq \operatorname{con}\left(a_{q}, b_{q}\right)$. Thus, $\operatorname{con}\left(x_{1}, y_{1}\right)=\operatorname{con}\left(a_{p}, b_{p}\right) \leq \operatorname{con}\left(a_{q}, b_{q}\right)=\operatorname{con}\left(x_{2}, y_{2}\right)$. This proves that $\mathcal{L}(H, I, J)$ satisfies $(\mathrm{C} 1)$.

Next, let $\alpha$ be the equivalence on $L(H, I, J)$ whose non-singleton equivalence classes are the $\left[a_{p}, b_{p}\right]$ for $p \in H^{-01}$, the $\left[c_{i}^{p q}, d_{i}^{p q}\right]$ for $\langle p, q\rangle \in I$ and $i \in$ $\{1, \ldots, 5\}$, and the $\left[c_{p q}^{i}, d_{p q}^{i}\right]$ for $\langle p, q\rangle \in J$ and $i \in\{1, \ldots, 5\}$. Using Lemma 4.1, it is straightforward to see that $\alpha$ is a congruence. Clearly, $\alpha$ is distinct from $\nabla_{L(H, I, J)}$. We claim that, for any $\langle x, y\rangle \in$ Pairs $^{\leq}(L(H, I, J))$,

$$
\gamma_{H, I, J}(\langle x, y\rangle)=1 \Longleftrightarrow \operatorname{con}(x, y)=\nabla_{L(H, I, J)} .
$$

To see this, assume that $\gamma_{H, I, J}(\langle x, y\rangle) \neq 1_{H}$. Then $\operatorname{con}(x, y) \leq \alpha$, defined in the paragraph above, and so $\operatorname{con}(x, y) \neq \nabla_{L(H, I, J)}$. This, together with (4.27), implies the validity of (4.28).

Next, in order to prove that $\mathcal{L}(H, I, J)$ satisfies $(\mathrm{C} 2)$, let us assume that $\left\langle u_{1}, v_{1}\right\rangle$ and $\left\langle u_{2}, v_{2}\right\rangle$ both belong to Pairs $\leq(L(H, I, J))$ such that $\operatorname{con}\left(u_{1}, v_{1}\right) \leq$ $\operatorname{con}\left(u_{2}, v_{2}\right)$. With the notation $p:=\gamma_{H, I, J}\left(\left\langle u_{1}, v_{1}\right\rangle\right)$ and $q:=\gamma_{H, I, J}\left(\left\langle u_{2}, v_{2}\right\rangle\right)$, we need to prove that $\langle p, q\rangle \in \nu_{H, I, J}$. Since, for $i \in\{1,2\}$,

$$
u_{i}=v_{i} \Longleftrightarrow \operatorname{con}\left(u_{i}, v_{i}\right)=\Delta_{L(H, I, J)} \Longleftrightarrow \gamma_{H, I, J}\left(u_{i}, v_{i}\right)=0,
$$

we can assume that $u_{1} \neq v_{1}, u_{2} \neq v_{2}$ and $p \neq 0 \neq q$. By (4.28), we can assume that $\operatorname{con}\left(u_{1}, v_{1}\right) \neq \nabla_{L(H, I, J)} \neq \operatorname{con}\left(u_{2}, v_{2}\right)$ and $p \neq 1 \neq q$. That is, $p, q \in H^{-01}$. Since $\left\langle u_{1}, v_{1}\right\rangle$ is projective to $\left\langle a_{p}, b_{p}\right\rangle$ by $(4.26), \operatorname{con}\left(u_{1}, v_{1}\right)=$ $\operatorname{con}\left(a_{p}, b_{p}\right)$. Furthermore, $\gamma_{H, I, J}\left(\left\langle u_{1}, v_{1}\right\rangle\right)=p=\gamma_{H, I, J}\left(\left\langle a_{p}, b_{p}\right\rangle\right)$ by (4.4). Hence, we can assume that $\left\langle u_{1}, v_{1}\right\rangle=\left\langle a_{p}, b_{p}\right\rangle$ and, similarly, $\left\langle u_{2}, v_{2}\right\rangle=\left\langle a_{q}, b_{q}\right\rangle$. After all these simplifications, in order to prove $(\mathrm{C} 2)$, we have to show that

$$
\begin{aligned}
& \text { if } p, q \in H^{-01}, \operatorname{con}\left(a_{p}, b_{p}\right) \leq \operatorname{con}\left(a_{q}, b_{q}\right) \neq \nabla_{L(H, I, J)}, \text { and } p \neq q, \\
& \text { then }\langle p, q\rangle=\left\langle\gamma_{H, I, J}\left(\left\langle a_{p}, b_{p}\right\rangle\right), \gamma_{H, I, J}\left(\left\langle a_{q}, b_{q}\right\rangle\right)\right\rangle \in \nu_{H, I, J} .
\end{aligned}
$$

By Lemma 4.2, there are covering pairs $\left\langle x_{i}, y_{i}\right\rangle \in \operatorname{Pairs}^{\prec}(L(H, I, J))$ such that

$$
\left\langle a_{q}, b_{q}\right\rangle=\left\langle x_{0}, y_{0}\right\rangle \stackrel{\mathrm{p} \text {-pr }}{\rightarrow}\left\langle x_{1}, y_{1}\right\rangle \stackrel{\mathrm{p} \text {-pr }}{\rightarrow} \cdots \stackrel{\text { p-pr }}{\rightarrow}\left\langle x_{n}, y_{n}\right\rangle=\left\langle a_{p}, b_{p}\right\rangle .
$$

We can assume that (4.30) is a shortest possible sequence and $n>0$. For $i=0, \ldots, n$, let $r_{i}=\gamma_{H, I, J}\left(\left\langle x_{i}, y_{i}\right\rangle\right)$. Of course, $r_{0}=q$ and $r_{n}=p$. Using appropriate initial or final segments of the sequence given in (4.30), the easy direction of Lemma 4.2 yields that $\operatorname{con}\left(a_{q}, b_{q}\right) \geq \operatorname{con}\left(x_{i}, y_{i}\right) \geq \operatorname{con}\left(a_{p}, b_{p}\right)$. 
Combining this with the premise in (4.29) and the definition of $\gamma_{H, I, J}$, we obtain that

$$
r_{i} \in H^{-01} \text { and }\{0,1\} \cap\left\{x_{i}, y_{i}\right\}=\varnothing, \quad \text { whenever } i \in\{0,1, \ldots, n\} .
$$

By the transitivity of $\nu_{H, I, J}$, it suffices to show that, for $i \in\{1, \ldots, n\}$,

$$
\left\langle r_{i}, r_{i-1}\right\rangle=\left\langle\gamma_{H, I, J}\left(\left\langle x_{i}, y_{i}\right\rangle\right), \gamma_{H, I, J}\left(\left\langle x_{i-1}, y_{i-1}\right\rangle\right)\right\rangle \in \nu_{H, I, J}
$$

By duality, we can assume that the $i$-th prime perspectivity in (4.30) is a prime-perspectivity down, that is, $\left\langle x_{i-1}, y_{i-1}\right\rangle \stackrel{\mathrm{p}-\mathrm{dn}}{\longrightarrow}\left\langle x_{i}, y_{i}\right\rangle$. We also assume that $r_{i} \neq r_{i-1}$, because otherwise (4.32) is trivial.

Since the sequence in (4.30) is of minimal length, $\left\langle x_{i-1}, y_{i-1}\right\rangle \neq\left\langle x_{i}, y_{i}\right\rangle$ and so $y_{i-1}>y_{i}$. We know that $L(H, I, J)$ is of length 5 , and (4.31) yields that

$$
1>y_{i-1}>y_{i} \succ x_{i}>0 \text {. }
$$

Hence, the interval $\left[y_{i}, y_{i-1}\right]$ is of length 1 or 2 .

First, assume that this interval is of length 2. The "zigzag structure" of our gadgets yield that $\left\langle x_{i-1}, y_{i-1}\right\rangle \stackrel{\mathrm{p}-\mathrm{dn}}{\rightarrow}\left\langle x_{i}, y_{i}\right\rangle$ cannot happen within a single gadget. Hence, there is an $s \in H^{-01}$ such both $\left\langle x_{i-1}, y_{i-1}\right\rangle$ and $\left\langle x_{i}, y_{i}\right\rangle$ are "thin edges" of appropriate basic gadgets, $\left\langle a_{s}, b_{s}\right\rangle$ is a common thin edge of these two gadgets, and $y_{i} \prec b_{s} \prec y_{i-1}$. However, then $r_{i}=s=r_{i-1}$; see Figures 6-9. This contradicts the assumption that $r_{i} \neq r_{i-1}$. Hence, $\left[y_{i}, y_{i-1}\right]$ is of length 1 , that is, $y_{i-1} \succ y_{i}$. It follows from the construction of $L(H, I, J)$ that both $\left\langle x_{i-1}, y_{i-1}\right\rangle$ and $\left\langle x_{i}, y_{i}\right\rangle$ are "thin edges" in the same basic gadget, and $\left\langle x_{i-1}, y_{i-1}\right\rangle \stackrel{\text { p-dn }}{\rightarrow}\left\langle x_{i}, y_{i}\right\rangle$ is only possible if $\left\langle x_{i-1}, y_{i-1}\right\rangle=\left\langle c_{5}^{r_{i} r_{i-1}}, d_{5}^{r_{i} r_{i-1}}\right\rangle$ and $\left\langle x_{i}, y_{i}\right\rangle=\left\langle e^{r_{i} r_{i-1}}, d_{4}^{r_{i} r_{i-1}}\right\rangle$. Hence, $G^{\mathrm{up}}\left(r_{i}, r_{i-1}\right)$ is present in $L(H, I, J)$, which means that $\left\langle r_{i}, r_{i-1}\right\rangle \in I$. Therefore, (4.24) gives that $\left\langle r_{i}, r_{i-1}\right\rangle \in \nu_{H, I, J}$, as required in (4.32).

Finally, if $I=J$, then $L(H, I, J)=L(H, I, I)$ is clearly a selfdual lattice, since we can obtain it from $L(H, \varnothing, \varnothing)$ by inserting only double gadgets. It is straightforward to see that the union of the polarity of $L(H, \varnothing, \varnothing)$ and the polarities of these double gadgets is a polarity $\pi$ of $L(H, I, I)$. Since $\pi$ preserves the quasi-coloring, $(\mathrm{C} 1)$ and $(\mathrm{C} 2)$ imply that $L(H, I, I)$ with this $\pi$ belongs to $\operatorname{PLat}_{(2.4)}^{\mathrm{emb}}$. This completes the proof of Lemma 4.6.

Next, with $\Theta_{\nu}$ defined right before (3.2), we formulate a corollary.

Lemma 4.7. Assuming (4.18), let $\mathcal{L}(H, I, J)$ be the quasi-colored lattice from Lemma 4.6, and let $\nu$ stand for the quasiordering $\nu_{H, I, J}$ from (4.24). Then the rule $[p] \Theta_{\nu} \mapsto \operatorname{con}\left(a_{p}, b_{p}\right)$ defines an order isomorphism

$$
\mu_{H, I, J}:\left\langle H / \Theta_{\nu} ; \nu / \Theta_{\nu}\right\rangle \rightarrow\langle\operatorname{Princ}(L(H, I, J)) ; \subseteq\rangle .
$$

Proof. To ease the notation in the proof, we omit $(H, I, J)$ from the notation. That is, we write $\mathcal{L}=\langle L, \leq ; \gamma ; H, \nu\rangle$ and $\mu$ instead of $(4.25)$ and $\mu_{H, I, J}$; then

$$
\mu:\left\langle H / \Theta_{\nu} ; \nu / \Theta_{\nu}\right\rangle \rightarrow\langle\operatorname{Princ}(L) ; \subseteq\rangle \text { is defined by }[p] \Theta_{\nu} \mapsto \operatorname{con}_{L}\left(a_{p}, b_{p}\right) .
$$


We need to show that $\mu$ is an order isomorphism. If $\left\langle[p] \Theta_{\nu},[q] \Theta_{\nu}\right\rangle \in \nu / \Theta_{\nu}$, then $\gamma\left(\left\langle a_{p}, b_{p}\right\rangle\right)=p \leq_{\nu} q=\gamma\left(\left\langle a_{q}, b_{q}\right\rangle\right)$, and (C1) implies that $\operatorname{con}_{L}\left(a_{p}, b_{p}\right) \leq$ $\operatorname{con}_{L}\left(a_{q}, b_{q}\right)$. Hence, $\mu$ is a well-defined map and it is order-preserving. Obviously, $\operatorname{Princ}(L)=\left\{\operatorname{con}_{L}(x, y):\langle x, y\rangle \in \operatorname{Pairs}^{\leq}(L)\right\}$. To prove that $\mu$ is surjective, let $\langle x, y\rangle$ belong to Pairs $\leq(L)$. With $r:=\gamma(\langle x, y\rangle)$, the equality $\gamma\left(\left\langle a_{r}, b_{r}\right\rangle\right)=r=\gamma(\langle x, y\rangle)$ and $(\mathrm{C} 1)$ imply that $\mu\left([r] \Theta_{\nu}\right)=\operatorname{con}_{L}\left(a_{r}, b_{r}\right)=$ $\operatorname{con}_{L}(x, y)$. Thus, $\mu$ is surjective. Finally, assume that $\mu\left([p] \Theta_{\nu}\right) \leq \mu\left([q] \Theta_{\nu}\right)$, that is, $\operatorname{con}_{L}\left(a_{p}, b_{p}\right) \leq \operatorname{con}_{L}\left(a_{q}, b_{q}\right)$. By $(\mathrm{C} 2), p=\gamma\left(\left\langle a_{p}, b_{p}\right\rangle\right) \leq_{\nu} \gamma\left(\left\langle a_{q}, b_{q}\right\rangle\right)=$ $q$, that is, $\left\langle[p] \Theta_{\nu},[q] \Theta_{\nu}\right\rangle \in \nu / \Theta_{\nu}$. This implies that $\mu$ is injective and $\mu^{-1}$ is order-preserving.

\section{Tailoring our quasi-colored lattices to the functor $F$}

In the rest of the paper, $F: \mathbf{C a t}(S) \rightarrow \mathbf{P O S}_{01}^{0 \text { s }}$ will be a functor as in Theorem 2.8. To ease the notation, we will write $\left\langle P_{i} ; \nu_{i}\right\rangle$, or $\left\langle P_{i} ; \leq_{i}\right\rangle$, and $\psi_{i j}$ instead of $F(i)$ and $F(i \leq j)$, respectively. The least element and the greatest element of $P_{i}$ are denoted by $0_{i}$ and $1_{i}$, respectively. We can assume that

$$
\text { for } i \neq j \in S, 0_{i}=0_{j}, 1_{i}=1_{j} \text {, and }\left|P_{i} \cap P_{j}\right|=2 \text {. }
$$

In the opposite case, we take two new elements, 0 and 1, outside $\bigcup\left\{P_{i}: i \in S\right\}$. Let $P_{i}^{\prime}=\left(P_{i} \backslash\left\{0_{i}, 1_{i}\right\}\right) \cup\{0,1\}$. We define an ordering $\leq_{i}^{\prime}$ on $P_{i}^{\prime}$ such that the map

$$
\alpha_{i}:\left\langle P_{i} ; \nu_{i}\right\rangle \rightarrow\left\langle P_{i}^{\prime} ; \nu_{i}^{\prime}\right\rangle, \text { defined by } x \mapsto \begin{cases}x, & \text { if } x \in P_{i} \backslash\left\{0_{i}, 1_{i}\right\}, \\ 0 & \text { if } x=0_{i}, \\ 1 & \text { if } x=1_{i},\end{cases}
$$

is an isomorphism. We let $\psi_{i j}^{\prime}=\alpha_{j} \circ \psi_{i j} \circ \alpha_{i}^{-1}$. Let $F^{\prime}: \operatorname{Cat}(S) \rightarrow \mathbf{P O S}_{01}^{0 \mathrm{~s}}$ be defined by $F^{\prime}(i)=\left\langle P_{i}^{\prime} ; \leq_{i}^{\prime}\right\rangle$ and $F(i \leq j)=\psi_{i j}^{\prime}$. This functor is naturally isomorphic to $F$, because $\alpha: F \rightarrow F^{\prime}$ is a natural isomorphism. Therefore, if (5.1) fails, then we can work with $F^{\prime}$ instead of $F$. This justifies assumption (5.1). For $j \in S$, let

$$
R_{j}:=\bigcup\left\{P_{i}: i \leq j\right\}
$$

Observe that $\nu_{i} \subseteq R_{j}^{2}:=R_{j} \times R_{j}, \psi_{i j} \subseteq R_{j}^{2}$ and $\psi_{i j}^{-1}=\left\{\langle x, y\rangle: x=\psi_{i j}(y)\right\} \subseteq$ $R_{j}^{2}$ for all $i \leq j$. Hence, we can let

$$
\begin{aligned}
\hat{\nu}_{j}=\operatorname{quo}_{R_{j}} & \left(\bigcup\left\{\nu_{i}: i \leq j, i \in S\right\}\right. \\
& \left.\cup \bigcup\left\{\psi_{i j}: i \leq j, i \in S\right\} \cup \bigcup\left\{\psi_{i j}^{-1}: i \leq j, i \in S\right\}\right) .
\end{aligned}
$$

Also, let $\hat{\Theta}_{j}=\hat{\nu}_{j} \cap \hat{\nu}_{j}^{-1}$. Note that as an easy consequence of $\psi_{i j}=\psi_{k j} \circ \psi_{i k}$,

$$
\text { for } i \leq k \leq j, \quad \psi_{i k} \subseteq \hat{\nu}_{j} \text { and } \psi_{i k}^{-1} \subseteq \hat{\nu}_{j}
$$


Lemma 5.1. For $j \in S$, the rule

$$
\kappa_{j}\left([x] \hat{\Theta}_{j}\right)= \begin{cases}x, & \text { if } x \in P_{j}, \\ \psi_{i j}(x), & \text { if } x \in P_{i},\end{cases}
$$

defines an order isomorphism $\kappa_{j}:\left\langle R_{j} / \hat{\Theta}_{j} ; \hat{\nu}_{j} / \hat{\Theta}_{j}\right\rangle \rightarrow\left\langle P_{j} ; \nu_{j}\right\rangle$.

The first line of (5.5) is only for emphasis; it can be omitted, since $\psi_{j j}$ is the identity map. Since $R_{j}=\bigcup\left\{P_{i}: i \leq j\right\}$ by definition, there exists an appropriate $i$ in the second line of (5.5). If $x \in P_{i_{1}} \cap P_{i_{2}}=\{0,1\}$, then no matter which of $i_{1}$ and $i_{2}$ serves as $i$, because $\psi_{i j}$ is $\{0,1\}$-preserving.

Proof. Consider the auxiliary map $\kappa_{j}^{\prime}:\left\langle R_{j} ; \hat{\nu}_{j}\right\rangle \rightarrow\left\langle P_{j} ; \nu_{j}\right\rangle$, defined by $\kappa_{j}^{\prime}(x):=$ $\psi_{i j}(x)$ for $x \in P_{i}$. This map is well defined, because $\psi_{i_{1} j}$ and $\psi_{i_{2} j}$ are not in conflict on $P_{i_{1}} \cap P_{i_{2}}=\{0,1\}$. First, we show that $\kappa_{j}^{\prime}$ is monotone in the sense that, for all $x, y \in R_{j}$,

$$
\text { if }\langle x, y\rangle \in \hat{\nu}_{j}, \quad \text { then }\left\langle\kappa_{j}^{\prime}(x), \kappa_{j}^{\prime}(y)\right\rangle \in \nu_{j} .
$$

By transitivity, it suffices to show this only for

$$
\langle x, y\rangle \in \bigcup\left\{\nu_{i}: i \leq j\right\} \cup \bigcup\left\{\psi_{i j}: i \leq j\right\} \cup \bigcup\left\{\psi_{i j}^{-1}: i \leq j\right\} ;
$$

see (5.3). If $\langle x, y\rangle \in \nu_{i}$ for some $i \leq j$, then $\left\langle\kappa_{j}^{\prime}(x), \kappa_{j}^{\prime}(y)\right\rangle=\left\langle\psi_{i j}(x), \psi_{i j}(y)\right\rangle$ belongs to $\nu_{j}$, because $\psi_{i j}$ is monotone. If $\langle x, y\rangle \in \psi_{i j}$, that is, $\psi_{i j}(x)=y$, then $\left\langle\kappa_{j}^{\prime}(x), \kappa_{j}^{\prime}(y)\right\rangle=\langle y, y\rangle \in \nu_{j}$ by reflexivity. Similarly, if $\langle x, y\rangle \in \psi_{i j}^{-1}$, that is, $\psi_{i j}(y)=x$, then $\left\langle\kappa_{j}^{\prime}(x), \kappa_{j}^{\prime}(y)\right\rangle=\langle x, x\rangle \in \nu_{j}$. This proves (5.6).

Note the rule $\kappa_{j}\left([x] \hat{\Theta}_{j}\right)=\kappa_{j}^{\prime}(x)$. If $[x] \hat{\Theta}_{j}=[y] \hat{\Theta}_{j}$, then $\langle x, y\rangle,\langle y, x\rangle \in \hat{\nu}_{j}$. So, (5.6) and the antisymmetry of $\nu_{j}$ yield that $\kappa_{j}^{\prime}(x)=\kappa_{j}^{\prime}(y)$. Hence, the map $\kappa_{j}$ from (5.5) is well defined. We also conclude from (5.6) that $\kappa_{j}$ is monotone. By the first line of $(5.5), \kappa_{j}$ is surjective. Hence, in order to complete the proof, it suffices to show that

$$
\text { if }\left\langle\kappa_{j}\left([x] \hat{\Theta}_{j}\right), \kappa_{j}\left([y] \hat{\Theta}_{j}\right)\right\rangle \in \nu_{j}, \quad \text { then }\left\langle[x] \hat{\Theta}_{j},[y] \hat{\Theta}_{j}\right\rangle \in \hat{\nu}_{j} / \hat{\Theta}_{j} ;
$$

note that the injectivity of $\kappa_{j}$ will follow from (5.7) since the ordering $\hat{\nu}_{j} / \hat{\Theta}_{j}$ is antisymmetric. In order to prove (5.7), assume that $\left\langle\kappa_{j}\left([x] \hat{\Theta}_{j}\right), \kappa_{j}\left([y] \hat{\Theta}_{j}\right)\right\rangle \in$ $\nu_{j}$. This means that $\left\langle\kappa_{j}^{\prime}(x), \kappa_{j}^{\prime}(y)\right\rangle \in \nu_{j}$, and we need to show that $\langle x, y\rangle \in \hat{\nu}_{j}$. By the definition of $R_{j}$, there are $i, k \in S$ with $i \leq j$ and $k \leq j$ such that $x \in P_{i}$ and $y \in P_{k}$. Hence, $\left\langle x, \kappa_{j}^{\prime}(x)\right\rangle=\left\langle x, \psi_{i j}(x)\right\rangle \in \psi_{i j} \subseteq \hat{\nu}_{j},\left\langle\kappa_{j}^{\prime}(x), \kappa_{j}^{\prime}(y)\right\rangle \in \nu_{j} \subseteq$ $\hat{\nu}_{j}$, and $\left\langle\kappa_{j}^{\prime}(y), y\right\rangle=\left\langle\psi_{k j}(y), y\right\rangle \in \psi_{k j}^{-1} \subseteq \hat{\nu}_{j}$ imply $\langle x, y\rangle \in \hat{\nu}_{j}$ by transitivity. This proves (5.7) and the lemma.

Proof of Theorem 2.8. First, we assume that $S$ has a largest element, $1 \in S$. Let $j \in S$. With $\hat{\nu}_{j}$ given in (5.3), we define

$$
I_{j}:=\left\{\langle x, y\rangle \in \hat{\nu}_{j}: 0 \neq x, 0 \neq y, x \neq y\right\} .
$$

Based on (4.21), we intend to define a functor $E: \mathbf{C a t}(S) \rightarrow \mathbf{L a t}_{\mathrm{sd} 5}^{\mathrm{emb}}$ as follows: $E(j):=L\left(R_{j}, I_{j}, I_{j}\right), \quad$ for $j \in S$,

$E(j \leq k):=$ the inclusion map $E(j) \rightarrow E(k), \quad$ for $j \leq k \in S$. 
We know from Lemma 4.6 that $E(j) \in \mathbf{L a t}_{\mathrm{sd} 5}^{\mathrm{emb}}$. To see that the second line of (5.9) makes sense, let $j \leq k \in S$. Combining (5.2), (5.3), (5.4), and (5.8), we have that $R_{j} \subseteq R_{k}$ and $I_{j} \subseteq I_{k}$. Hence, by (4.22), $E(j)$ is a sublattice of $E(k)$. Thus, $E$ from (5.9) is a functor. Let $L=E(1)$. By (4.22), all $E(j)$, for $j \in S$, are sublattices of $L$. Actually, they are $\{0,1\}$-sublattices, because we know from Lemma 4.6 that both $L$ and the $E(j)$ are of length 5 . Hence, (i) and (ii) of Definition 2.7 are satisfied. Assume, for a moment, that $s, t \in S$ such that $s \not \leq t$. Then $R_{s} \nsubseteq R_{t}$ by (5.1) and (5.2), $L\left(R_{s}, \varnothing, \varnothing\right) \nsubseteq L\left(R_{t}, \varnothing, \varnothing\right)$ by (4.16), and so $E(s)=L\left(R_{s}, I_{s}, I_{s}\right) \nsubseteq L\left(R_{t}, I_{t}, I_{t}\right)=R(t)$. Thus, Definition 2.7(iii) holds, and it suffices to prove that $E$ lifts $F$ with respect to Princ. Next, we claim that

$$
\hat{\nu}_{j}=\nu_{R_{j}, I_{j}, I_{j}} .
$$

We know from (4.24) that

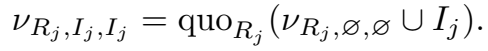

If $\langle x, y\rangle \in \nu_{R_{j}, \varnothing, \varnothing}$ and $x \neq y$, then $\langle x, y\rangle=\langle 0, p\rangle$ or $\langle x, y\rangle=\langle p, 1\rangle$ for some $p \in R_{j}^{-01}$ by (4.23). By (5.1) and (5.2), we have that $p \in P_{i}$ and $\langle x, y\rangle \in \nu_{i}$

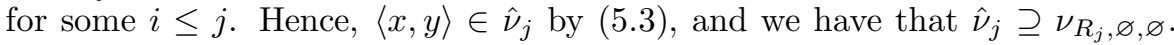
Since $\hat{\nu}_{j} \supseteq I_{j}$ also holds by (5.8), (5.11) yields that $\hat{\nu}_{j} \supseteq \nu_{R_{j}, I_{j}, I_{j}}$. In order to prove the converse inclusion for (5.10), assume that $\langle x, y\rangle$ belongs to the union in (5.3) and $x \neq y$; we need to show that $\langle x, y\rangle \in \nu_{R_{j}, I_{j}, I_{j}}$. We can assume that $x \neq 0 \neq y$, since otherwise $\langle x, y\rangle \in \hat{\nu}_{j}$ would easily give that $\langle x, y\rangle \in$ $I_{j} \subseteq \nu_{R_{j}, I_{j}, I_{j}}$ by (5.8) and (5.11). If $x=0$, then $\langle x, y\rangle \in \nu_{R_{j}, \varnothing, \varnothing \subseteq} \subseteq \nu_{R_{j}, I_{j}, I_{j}}$ by (4.23) and (5.11). If $y=0$, then $x=0$, because (5.3) gives that for some $i \leq j$, either $\langle x, 0\rangle \in \nu_{i}$ and 0 is the unique least element of the ordered set $\left\langle P_{i} ; \nu_{i}\right\rangle$, or $\langle x, 0\rangle \in \psi_{i j}$ and $x=0$ since $\psi_{i j}$ is 0 -separating, or $\langle x, 0\rangle \in \psi_{i j}^{-1}$ and $x=0$ since $\psi_{i j}$ is 0 -preserving. So if $y=0$, then $\langle x, y\rangle=\langle 0,0\rangle \in \nu_{R_{j}, I_{j}, I_{j}}$ by reflexivity. In this way, we have shown that $\hat{\nu}_{j} \subseteq \nu_{R_{j}, I_{j}, I_{j}}$. That is, (5.10) holds.

Armed with (5.10) and writing $\hat{\nu}_{j}, \mu_{j}$, and $\hat{\Theta}_{j}$ instead of $\nu_{R_{j}, I_{j}, I_{j}}, \mu_{R_{j}, I_{j}, I_{j}}$, and $\Theta_{\nu_{R_{j}, I_{j}, I_{j}}}$, respectively, Lemma 4.7 yields that

$$
\begin{array}{r}
\mu_{j}:\left\langle R_{j} / \hat{\Theta}_{j} ; \hat{\nu}_{j} / \hat{\Theta}_{j}\right\rangle \rightarrow\langle\operatorname{Princ}(E(j)) ; \subseteq\rangle, \\
\text { defined by }[p] \hat{\Theta}_{j} \mapsto \operatorname{con}_{E(j)}\left(a_{p}, b_{p}\right),
\end{array}
$$

is an order isomorphism. So is $\kappa_{j}$ from Lemma 5.1 . Hence, the composite map

$$
\xi_{j}=\mu_{j} \circ \kappa_{j}^{-1}, \text { from } F(j)=\left\langle P_{j} ; \nu_{j}\right\rangle \text { to }(\operatorname{Princ} \circ E)(j)=\langle\operatorname{Princ}(E(j)) ; \subseteq\rangle \text {, }
$$

is also an order isomorphism. In order to show that $\xi$, defined by $\xi(j)=\xi_{j}$, is a natural isomorphism from $F$ to Princ $\circ E$, we need to prove that, for 
$j \leq k \in S$, the diagram

$$
\begin{array}{ccc}
\left\langle P_{j} ; \nu_{j}\right\rangle & \stackrel{\psi_{j k}}{\longrightarrow} & \left\langle P_{k} ; \nu_{k}\right\rangle \\
\xi_{j} \downarrow & \xi_{k} \downarrow \\
\langle\operatorname{Princ}(E(j)) ; \subseteq\rangle & \stackrel{\zeta_{E(j), E(k)}}{\longrightarrow}\langle\operatorname{Princ}(E(k)) ; \subseteq\rangle
\end{array}
$$

commutes, because the lower arrow is (Princ $\circ E)(j \leq k)$ by Remark 2.4 and $\psi_{j k}=F(j \leq k)$. To do so, consider an arbitrary element $p \in P_{j}$. By (5.5), $\kappa_{j}\left([p] \hat{\Theta}_{j}\right)=p$. Thus, (5.12) yields that $\xi_{j}(p)=\mu_{j}\left(\kappa_{j}^{-1}(p)\right)=\operatorname{con}_{E(j)}\left(a_{p}, b_{p}\right)$. Consequently,

$$
\zeta_{E(j), E(k)}\left(\xi_{j}(p)\right)=\zeta_{E(j), E(k)}\left(\operatorname{con}_{E(j)}\left(a_{p}, b_{p}\right)\right)=\operatorname{con}_{E(k)}\left(a_{p}, b_{p}\right) .
$$

Using (5.5) again, $\kappa_{k}\left([p] \hat{\Theta}_{k}\right)=\psi_{j k}(p)$. Hence, $\kappa_{k}^{-1}\left(\psi_{j k}(p)\right)=[p] \hat{\Theta}_{k}$. Thus,

$$
\xi_{k}\left(\psi_{j k}(p)\right)=\mu_{k}\left(\kappa_{k}^{-1}\left(\psi_{j k}(p)\right)\right)=\mu_{k}\left([p] \hat{\Theta}_{k}\right)=\operatorname{con}_{E(k)}\left(a_{p}, b_{p}\right) .
$$

Finally, we conclude from (5.14) and (5.15) that (5.13) is a commutative diagram. Therefore, $\xi$ is a natural isomorphism and Definition 2.7(iv) holds, that is, $E$ lifts $F$ with respect to Princ.

Second, assume that $1 \notin S$. Add 1 as a new top to $S$ to obtain $S_{1}=S \cup\{1\}$. Extend $F$ to a functor $F_{1}$ : $\mathbf{C a t}\left(S_{1}\right) \rightarrow \mathbf{P O S}_{01}^{0 \mathrm{~s}}$ by letting $F_{1}(1)=\{0,1\}$, the two-element chain, and defining $F_{1}(i \leq 1)=\psi_{i 1}: F_{1}(i) \rightarrow F_{1}(1)$ by the rule $\psi_{i 1}(x)=0 \Longleftrightarrow x=0$. Clearly, $F_{1}$ is a functor from $\operatorname{Cat}\left(S_{1}\right)$ to $\mathbf{P O S}_{01}^{0 \mathrm{~s}}$. Since it is concretely representable by the first part of the proof, so is its restriction, $F$.

Finally, we have already seen that $E(j)=L\left(R_{j}, I_{j}, I_{j}\right)$ belongs to $\mathbf{P L a t}_{(2.4)}^{\mathrm{emb}}$. Clearly, the inclusion map $E(j \leq k)$ from (5.9) is polarity-preserving. This completes the proof of Theorem 2.8 .

\section{Concluding remarks}

Remark 6.1. In order to construct smaller lattices, we can replace the $I_{j}$ in (5.8) by appropriate subsets $I_{j}^{\prime}$ such that quo $\left(\nu_{R_{j}, \varnothing, \varnothing} \cup I_{j}^{\prime}\right)=\operatorname{quo}\left(\nu_{R_{j}, \varnothing, \varnothing} \cup I_{j}\right)$, see (4.24), and $I_{j}^{\prime} \subseteq I_{k}^{\prime}$ for $j \leq k$. Note that Examples 2.2 and 3.1 use this simplification; this is why, say, there is no arrow between $\left\langle a_{q_{3}}, b_{q_{3}}\right\rangle$ and $\left\langle a_{r_{3}}, b_{r_{3}}\right\rangle$ in $W_{1}$ of Figure 3 .

Remark 6.2. In order to reduce the sizes of our lattices even further, let $G_{-e}^{\mathrm{db}}(p, q)$ denote the lattice that we obtain from $G^{\mathrm{db}}(p, q)$ by omitting $e^{p q}$ and $e_{p q}$. As an ordered set, $G_{-e}^{\mathrm{db}}(p, q)$ is a lattice, though not a sublattice of $G^{\mathrm{db}}(p, q)$. To keep our proof simple, we used both $G^{\mathrm{db}}(p, q)$ and $G^{\mathrm{db}}(q, p)$ to force that $\operatorname{con}\left(a_{p}, b_{p}\right)=\operatorname{con}\left(a_{q}, b_{q}\right)$. However, we can use $G_{-e}^{\mathrm{db}}(p, q)$ alone for this purpose; then (3.4) should be disregarded, because $\left|G_{-e}^{\mathrm{db}}\right|=20<22=$ $\left|G^{\mathrm{db}}\right|$. 
Example 6.3 (Continuation of Examples 2.2 and 3.1). Based on Remark 6.2, we can obtain smaller lattices as follows. For each dotted arc in Figure 3, we insert a copy of $G_{-e}^{\mathrm{db}}$, which brings 20 new elements. For the solid edge, we insert $G^{\mathrm{db}}\left(q_{3}, p_{3}\right)$, which adds 22 new elements. If $L_{0}^{\prime}, \ldots, L_{3}^{\prime}$ denote the lattices we obtain in this way, then $\left|L_{1}^{\prime}\right|=14+14+6 \cdot 20+22=170$. Similarly, $\left|L_{0}^{\prime}\right|=14+2=16,\left|L_{2}\right|=14+4+20=38$, and $\left|L_{3}\right|=14+8+20+22=64$.

6.1. Added on May 4, 2016. An anonymous referee has pointed out that the argument of F. Wehrung [30, Sect. 7-4.5] implies that we cannot replace $\operatorname{Cat}(S)$ in Theorem 2.8 with an arbitrary small category. Actually, the same holds even if we take the category Lat of all lattices with all lattice homomorphisms rather than $\mathbf{L a t}_{\mathrm{sd} 5}^{\mathrm{emb}}$. We demonstrate this with the following example.

Example 6.4. Let $A$ and $B$ be the two-element chain and the three-element chain, respectively. They belong to $\mathbf{P O S}_{01}^{0 \mathrm{~s}}$. Let $e: A \rightarrow B$ and $p: B \rightarrow A$ be the unique $\mathbf{P O S}_{01}^{0 \text { s }}$-morphisms between $A$ and $B$. The set $\{A, B\}$ of objects and the set $\left\{\operatorname{id}_{A}, \operatorname{id}_{B}, e, p, e \circ p\right\}$ of morphisms constitute a small category $\mathbf{C}$, which is a full subcategory of $\mathbf{P O S}_{01}^{0 \mathrm{~s}}$. Let $F: \mathbf{C} \rightarrow \mathbf{P O S}_{01}^{0 \mathrm{~s}}$ be the inclusion functor; that is, $F(x)=x$ for all $x \in\left\{A, B, \mathrm{id}_{A}, \mathrm{id}_{B}, e, p, e \circ p\right\}$.

The meaning of "in Lat" below is self-explanatory by Definition 2.6.

Observation 6.5 (Suggested by an anonymous referee). F from Example 6.4 is not representable by principal lattice congruences in Lat.

Proof. For the sake of contradiction, suppose that $E: \mathbf{C} \rightarrow$ Lat lifts $F$ with respect to Princ. By Definition 2.6, there exists a natural isomorphism $\xi: F \rightarrow$ Princ $\circ E$. In particular, since $F$ acts identically, the diagram

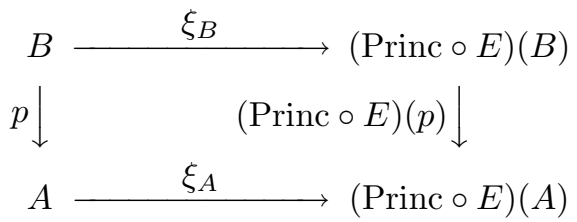

commutes. Hence, (Princ $\circ E)(p)=\xi_{A} \circ p \circ \xi_{B}^{-1}$. Since all the three factors are 0 -separating, so is $\operatorname{Princ}(E(p))=(\operatorname{Princ} \circ E)(p)$. If $x, y \in E(B)$ such that $E(p)(x)=E(p)(y)$, then $(2.3)$ yields that

$$
\operatorname{Princ}(E(p))\left(\operatorname{con}_{E(B)}(x, y)\right)=\operatorname{con}_{E(A)}(E(p)(x), E(p)(y))=\Delta_{E(A)} .
$$

Thus $\operatorname{con}_{E(B)}(x, y)=0_{\operatorname{Princ}(E(B))}=\Delta_{E(B)}$, since Princ $(E(p))$ is 0-separating, and we have that $x=y$. Consequently, $E(p)$ is injective. Since $E$ is a functor and $\mathrm{id}_{A}$ is an identity morphism in $\mathbf{C}, E(p) \circ E(e)=E(p \circ e)=E\left(\mathrm{id}_{A}\right)=$ $\operatorname{id}_{E(A)}$. Therefore, $E(p)$ is surjective and so it is an isomorphism in Lat. It follows that $\operatorname{Princ}(E(p))=(\operatorname{Princo} E)(p)$ is an isomorphism in $\mathbf{P O S}_{01}^{0 \mathrm{~s}}$. Finally, since $p=\xi_{A}^{-1} \circ($ Princ $\circ E)(p) \circ \xi_{B}$ by the commutativity of (6.1) and each of these three factors is an isomorphism, $p$ is an isomorphism in $\mathbf{P O S}_{01}^{0 \mathrm{~s}}$. This contradicts the definition of $p$ (and $|A| \neq|B|$ ), completing the proof. 
The following remark is needed in G. Czédli [4].

Remark 6.6. It is clear from (3.3) and the last sentence of the proof of Lemma 4.5 that $M_{4 \times 3}$ can be replaced by any simple selfdual lattice having at least four elements; then Lemmas 4.6 and 4.7 remain true except that the length of $L(H, I, J)$ need not be 5 . For every $p \in H$ and $x, y \in L(H, I, J)$, if $x<a_{p}$ and $b_{p}<y$, then both $\left\langle x, a_{p}\right\rangle$ and $\left\langle b_{p}, y\right\rangle$ are 1-colored by our construction, and each of (4.28) and Lemma 4.7 implies that $\operatorname{con}\left(x, a_{p}\right)=$ $\nabla_{L(H, I, J)}=\operatorname{con}\left(b_{p}, y\right)$. Finally, due to some last minute change in the present paper, where [4] references (4.23), it should be understood as (4.24).

Acknowledgment. The referees' hints are highly appreciated; some of these hints are mentioned in Subsections 2.3 and 6.1.

\section{REFERENCES}

[1] Bogart, K.P., Freese, R., Kung, J.P.S. (eds): The Dilworth Theorems. Selected papers of Robert P. Dilworth. Birkhäuser, Boston (1990)

[2] Czédli, G.: Representing homomorphisms of distributive lattices as restrictions of congruences of rectangular lattices. Algebra Universalis 67, 313-345 (2012)

[3] Czédli, G.: Representing a monotone map by principal lattice congruences. Acta Math. Hungar. 147, 12-18 (2015)

[4] Czédli, G.: An independence theorem for ordered sets of principal congruences and automorphism groups of bounded lattices. Acta Sci. Math. 82, 3-18 (2016)

[5] Czédli, G.: The ordered set of principal congruences of a countable lattice. Algebra Universalis 75, 351-380 (2016)

[6] Czédli, G.: Diagrams and rectangular extensions of planar semimodular lattices. Algebra Universalis, submitted; arXiv: 1412.4453

[7] Czédli, G., Schmidt, E.T.: Finite distributive lattices are congruence lattices of almost-geometric lattices. Algebra Universalis 65, 91-108 (2011)

[8] Funayama, N., Nakayama, T.: On the distributivity of a lattice of lattice-congruences. Proc. Imp. Acad. Tokyo 18, 553-554 (1942)

[9] Gillibert, P.; Wehrung, F.: From objects to diagrams for ranges of functors. Lecture Notes in Mathematics 2029. Springer, Heidelberg (2011)

[10] Grätzer, G.: The Congruences of a Finite Lattice. A Proof-by-picture Approach. Birkhäuser, Boston (2006)

[11] Grätzer, G.: Lattice Theory: Foundation. Birkhäuser, Basel (2011)

[12] Grätzer, G.: The order of principal congruences of a bounded lattice. Algebra Universalis 70, 95-105 (2013)

[13] Grätzer, G.: A technical lemma for congruences of finite lattices. Algebra Universalis 72, 53-55 (2014)

[14] Grätzer, G.: Congruences and prime-perspectivities in finite lattices. Algebra Universalis 74, 351-359 (2015)

[15] Grätzer, G., Knapp, E.: Notes on planar semimodular lattices. III. Congruences of rectangular lattices. Acta Sci. Math. (Szeged) 75, 29-48 (2009)

[16] Grätzer, G., Lakser, H.: Homomorphisms of distributive lattices as restrictions of congruences. Canad. J Math. 38, 1122-1134 (1986)

[17] Grätzer, G., Lakser, H.: Homomorphisms of distributive lattices as restrictions of congruences. II. Planarity and automorphisms. Canad. J Math. 46, 3-54 (1994)

[18] Grätzer, G., Lakser, H.: Representing homomorphisms of congruence lattices as restrictions of congruences of isoform lattices. Acta Sci. Math. (Szeged) 75, 393-421 (2009)

[19] Grätzer, G., Lakser, H., Schmidt, E.T.: Isotone maps as maps of congruences. I. Abstract maps. Acta Math. Hungar. 75, 105-135 (1997) 
[20] Grätzer, G., Lakser, H., Schmidt, E.T.: Congruence lattices of finite semimodular lattices. Canad. Math. Bull. 41, 290-297 (1998)

[21] Grätzer, G., Lakser, H., Schmidt, E.T.: Isotone maps as maps of congruences. II. Concrete maps. Acta Math. Hungar. 92, 233-238 (2001)

[22] Grätzer, G., Schmidt, E.T.: On congruence lattices of lattices. Acta Math. Acad. Sci. Hungar. 13, 179-185 (1962)

[23] Grätzer, G., Schmidt, E.T.: An extension theorem for planar semimodular lattices. Period. Math. Hungar. 69, 32-40 (2014)

[24] Huhn, A. P.: On the representation of distributive algebraic lattices. III. Acta Sci. Math. (Szeged) 53, 11-18 (1989)

[25] Kamara, M.: Zur Konstruktion vollständiger Polaritätsverbände. (German) J. Reine Angew. Math. 299/300, 280-286 (1978)

[26] Kelly, D., Rival, I.: Planar lattices. Canad. J. Math. 27, 636-665 (1975)

[27] Nation, J. B.: Notes on Lattice Theory. http://www .math.hawaii.edu/ jb/books.html

[28] Růžička, P.: Free trees and the optimal bound in Wehrung's theorem. Fund. Math. 198, 217-228 (2008)

[29] Wehrung, F.: A solution to Dilworth's congruence lattice problem. Adv. Math. 216 610-625 (2007)

[30] Wehrung, F.: Schmidt and Pudlák's approaches to CLP. In: Grätzer, G., Wehrung, F (eds.) Lattice Theory: Special Topics and Applications I, pp. 235-296. Birkhäuser, Basel (2014)

\section{GÁBor CzÉDli}

University of Szeged, Bolyai Institute, Szeged, Aradi vértanúk tere 1, HUNGARY 6720 e-mail: czedli@math.u-szeged.hu URL: http://www.math.u-szeged.hu/ czedli/ 